\title{
Won't You Think of Others? Interdependent Privacy in Smartphone App Permissions
}

\author{
MAXIMILIAN MARSCH, Technical University of Munich, Germany \\ JENS GROSSKLAGS, Technical University of Munich, Germany \\ SAMEER PATIL, School of Computing, University of Utah, United States
}

\begin{abstract}
The ever increasing amount of data on smartphones often contains private information of others that people interact with via the device. As a result, one user's decisions regarding app permissions can expose the information of other parties. However, research typically focuses on consequences of privacy-related decisions only for the user who makes the decisions. Work on the impact of these decisions on the privacy of others is still relatively scant. We fill this gap with an online study that extends prior work on interdependent privacy in social networking sites to the context of smartphone permissions. Our findings indicate that people typically give less consideration to the implications of their actions for the privacy of others compared to the impact on themselves. However, we found that priming people with information that features others can help reduce this discrepancy. We apply this insight to offer suggestions for enhancing permission-specification interfaces and system architectures to accommodate interdependent privacy.
\end{abstract}

CCS Concepts: • Human-centered computing $\rightarrow$ Empirical studies in HCI; Empirical studies in ubiquitous and mobile computing; Smartphones; • Security and privacy $\rightarrow$ Social aspects of security and privacy.

Additional Key Words and Phrases: Interdependent privacy, multiparty privacy conflicts, smartphone app permissions, calendar, contacts, photos

ACM Reference Format:

Maximilian Marsch, Jens Grossklags, and Sameer Patil. 2021. Won't You Think of Others? Interdependent Privacy in Smartphone App Permissions. Proc. ACM Hum.-Comput. Interact. 5, CSCW2, Article 437 (October 2021), 35 pages. https://doi.org/10.1145/3479581

\section{INTRODUCTION}

The ever increasing amount of data on people's smartphones often contains private information of others that people interact with via the device. As a result, one user's decisions regarding app permissions can expose the information of other users, thus violating their privacy. For instance, social media companies have been known to create profiles for those who do not even use their services, based simply on the information revealed by the practices of their friends who do use social media.

Previous work in this area approaches the problem with the basic insight that information may be jointly owned, potentially leading to conflict and associated negotiation [53]. Examination of this issue has been labeled as multi-party privacy (MPC) [69], collaborative privacy [46], peer privacy [13], or networked privacy [12]. Within this space, interdependent privacy scenarios capture situations where "the actions of some individuals affect the privacy of other individuals" [11].

Authors' addresses: Maximilian Marsch, Technical University of Munich, Munich, Germany, maximilian.marsch@tum.de; Jens Grossklags, Technical University of Munich, Munich, Germany, jens.grossklags@in.tum.de; Sameer Patil, School of Computing, University of Utah, Salt Lake City, Utah, 84112, United States, sameer.patil@utah.edu.

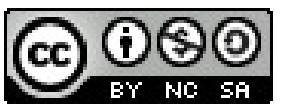

This work is licensed under a Creative Commons Attribution-NonCommercial-ShareAlike International 4.0 License.

(C) 2021 Copyright held by the owner/author(s).

2573-0142/2021/10-ART437. https://doi.org/10.1145/3479581

Proc. ACM Hum.-Comput. Interact., Vol. 5, No. CSCW2, Article 437. Publication date: October 2021. 
Yet, in comparison to the vast body of privacy research devoted to consequences of privacyrelated decisions for those who make decisions [3], work on the impact of these decisions on the privacy of others is still comparatively scant [31]. Moreover, much of the research that tackles the topic of interdependent privacy and consequent conflicts in privacy preferences has been carried out in the context of Social Network Sites (SNS) (e.g., $[35,57]$ ). However, there are important operational differences between SNS and smartphones. In particular, SNS are designed for information sharing and social interactions, many of which are (semi-)public. Further, SNS users must explicitly approve their connections to others, thus allowing people some degree of control over their associations with other parties. For instance, as a recourse for protection against the actions of others, an SNS user may choose to deny a connection request or block an existing contact.

In contrast, smartphones are typically single-user personal devices that nonetheless contain potentially sensitive information about parties other than the user. Such information can be present in the form of photos, contact information, calendar invites, and so on. One is typically unaware of the presence of one's information on other people's smartphones and powerless to influence the decisions of other users to share that information with the apps on their smartphones. As a result, in the smartphone context, one must rely exclusively on the judgment and discretion of other people for protecting the privacy of one's personal information present on their devices.

The literature on interdependent privacy in the SNS context indicates that people tend to pay less attention to the privacy of other people's data in comparison to their own [55], and research on individual privacy decision making underscores that people often share private information for even minor conveniences and rewards [4]. Moreover, prior work on interdependent privacy, conflicting preferences, and permission choices has often focused on a specific data type (e.g., photos) or a specific permission (e.g., location). There is a need to verify the extent to which findings pertaining to interdependent privacy in SNS apply to the smartphone context and how they might differ based on data type. Mechanisms that can help surface the impact of a user's privacy choices on the user's professional and personal connections need to be studied as well.

We address the above aspects via the following research questions:

RQ1: Do individuals consider the privacy of other people when making decisions pertaining to smartphone app permissions?

RQ2: Does presenting information cues that feature other people prime users to give greater consideration to the privacy of others when making decisions pertaining to smartphone app permissions?

RQ3: Do concerns for privacy of other people vary based on the type of permission (specifically, calendar, contacts, and photos) requested by a smartphone app?

To answer these questions, we developed a scenario-based between-subjects study with six conditions (three app permissions $\mathrm{x}$ two types of priming). We deployed the study as an online questionnaire via the Amazon Mechanical Turk (AMT) crowdwork platform. Based on the presented scenario, participants answered questions related to privacy for self and others for smartphone permissions for app access to calendar, contacts, or photos. For each of these permissions, we presented a corresponding screenshot of information with the presence or absence of other people. Based on comparing the results between the six conditions, we make the following contributions:

- We extend findings pertaining to interdependent privacy to the context of smartphone apps.

- We show that presenting information featuring other people can prime people to give more consideration to the privacy of others.

- We apply our insight to propose recommendations for improving the user interface (UI) and system design that could help users act in ways that minimize the impact of their actions on the privacy of others (as well as their own). 
In the sections that follow, we first situate our work in the literature. Next, we describe our method in detail, followed by the findings of the statistical analyses of the collected data. We then discuss the insight gained from the findings and apply it to generate enhancements for system design that can help tackle interdependent privacy issues in the smartphone context. We conclude by offering promising directions for future research.

\section{RELATED WORK}

Our research builds on prior work on interdependent privacy and access permissions on mobile devices. We discuss each in turn.

\subsection{Interdependent Privacy Research}

Our work falls within the conceptual domain of interdependent privacy. Biszók and Chia [11] define interdependent privacy scenarios as those where "the actions of some individuals affect the privacy of other individuals." In practice, interdependent privacy issues arise because of a variety of sharing decisions in SNS and beyond. Permission systems can make these potential conflicts particularly visible. For example, in the Facebook context, permissions relate not only to a friend's basic information, such as hometown, but also cover more sensitive data, such as birth date, location, photos and videos, etc. [77]. Even if such information is not used pervasively by a majority of apps, the impact of permitting access to the information is amplified by the simple fact that with each sharing decision, data of hundreds, if not thousands, of users is shared in bulk.

From a theoretical perspective, interdependent privacy has been studied in various game-theoretic scenarios, such as $2 \times 2$ player games [11] and networks [54, 71]. The results of these theoretical explorations show that rational users have incentives to share other people's data in a variety of scenarios. Studies with an empirical approach provide complementary insight on interdependent privacy based on surveys and analytics. Krasnova et al. [35] did not find a strong difference between the level of concern about permissions related to one's own data vs. that of one's friends. Yet, several studies by $\mathrm{Pu}$ and Grossklags [55-57] indicate that users value their own information considerably more than the information of others regarding whom they make sharing decisions. Likewise, qualitative research (e.g., [63]) has documented that people express concerns about their data being shared by other parties. Further, Amon et al. [8] showed that explicitly priming individuals to consider the privacy of others may lead to even more disclosure of other people's data (such as photos). To counter the interdependent privacy threats highlighted in the theoretical and empirical works, researchers have recently begun exploring technical solutions. For example, recent efforts by Hasan et al. [27] and Dimiccoli et al. [20] leverage machine learning to blur bystanders in photos.

Interdependent privacy has been studied only over the last ten years. Humbert et al. [31] provide a detailed review of the extant literature and the different related concepts. Closely related to interdependent privacy is the notion of networked privacy that highlights the data practices that affect persons beyond the sharer [12]. Peer privacy or peer disclosure relates to situations in which private information about an individual is shared by others [13]. When privacy can be influenced by multiple parties, it can lead to multi-party privacy conflicts (MPC) $[68,69,73]$ as well as collaborative privacy issues $[46,67]$. The latter arise in cases in which individuals perceive information to be owned and shared in a joint fashion, triggering a negotiation process about mutually agreeable disclosure [53]. Sharing decisions about other people's information are further governed by norms and contextual factors [41].

Most research on interdependent privacy and related concepts has been undertaken in the SNS context. A few studies have covered interdependent privacy issues that arise in a number of other domains, such as Smart Homes [23, 85] and shared data storage in the cloud [81]. However, we are unaware of research on interdependent privacy in the context of data on people's smartphones. We 
aim to shed light on these matters as they pertain to smartphone app permissions. By doing so, we extend the literature on interdependent privacy as well as work on preferences and practices regarding individual privacy when specifying smartphone permissions (e.g., [22, 33]).

\subsection{Mobile-Device Permission Systems}

In contrast to typical permission systems offered by SNS, the mobile app context currently makes no clear distinction between permissions relating primarily to user data (e.g., the user's birthday) vs. data about others (e.g., the birthdays of a user's contacts). However, by their very nature, several permissions on mobile devices can expose data about others, such as contacts, photos, call history, calendar, etc. Requesting such permissions is quite popular in the mobile app ecosystem. As early as 2014, a large-scale study of Android apps found that $10 \%$ of the surveyed apps requested contact lists, with a few even asking for call histories [32]. Likewise, Zscaler demonstrated that popular iOS apps frequently request access to contacts (92\%) or calendars (32\%) [60]. A 2020 survey of 1,000 important Australian apps reported that access to contacts was requested by 218 apps, calendar information was demanded by 73 apps, and READ_EXTERNAL_STORAGE (which is, for example, necessary to access photo files) was requested by 611 apps in the sample [9].

We found no prior work on mobile permissions with an explicit focus on interdependent privacy. However, permissions affecting other people's data have often been a part of broader investigations. For example, Porter Felt et al. [22] found that many people would be "very upset" if apps shared their photos $(90.6 \% ; 81.3 \%)$, calendar $(83.7 \% ; 63.6 \%)$, or contacts (not asked; $73.6 \%$ ) publicly [first value] or with their friends [second value]. Many investigations have focused on the important distinction between 'normal' and 'dangerous' permissions [1, 65]. For example, several research studies have reported that users struggle to avoid misuse of sensors (such as camera, microphone, etc.) by malicious apps $[51,52]$. However, these studies on granting or withholding of individual permissions involve no explicit consideration of interdependent privacy.

Over-privileged apps that request more permissions than strictly necessary for the app's purpose is another core problem in the space of mobile apps. For example, Porter Felt et al. [21] found that over 35\% of a sample of 900 Android apps requested unneeded permissions. Google researchers notified app developers whose apps used more permissions than functionally-comparable apps, leading these developers to reduce the number of permissions significantly more than those from a control group [49]. Nudging can be effective for the individual users as well. Almuhimedi et al. [7] showed that using a permissions manager that delivers notifications about app behavior can lead users to revisit app settings and disable permissions they deem inappropriate. Harbach et al. [26] proposed the use of personal examples to influence user behavior, triggering more risk-aware choices about app adoption but also heightening negative affect. A number of efforts have gone a step further and demonstrated that machine learning can be applied to manage smartphone permissions on behalf of the user [39, 43, 75, 82]. Such automated approaches can be helpful because several studies demonstrate that users typically do not understand the complex system of permissions offered by mobile operating systems and are often ignorant of the consequences of their permission choices [22, 33, 50, 58].

While the research space around mobile devices and their permission systems is rich and diverse, we are unaware of research focusing on interdependent privacy issues in this domain. In particular, our work is focused on understanding whether users protect the privacy of the data that they gather and hold about others on their increasingly powerful devices.

\section{METHOD}

The following subsections describe the study procedures and the participant sample. The Technical University of Munich does not require ethics approval for questionnaire-based online studies. 
However, when conducting the study and analyzing the data, we followed standard practices for ethical research: presenting detailed study procedures, obtaining consent, not collecting identifiable information or device data, and using a platform compliant with the European Union's General Data Protection Regulation (GDPR). The instruments used for the study are provided in Appendix B.

\subsection{Research Design}

We designed a 3x2 study that systematically varied three smartphone permissions (calendar, contacts, and photos) and two information presentation modes (Non-persons and Persons). To that end, we employed a scenario involving a fictitious person named Thomas to generate separate vignettes for each of the three permissions:

Thomas is an undergraduate student at his local University. He is looking for a new app for his smartphone. After some searching, he found an app which he installed on his phone without hesitation.

This app asked for access to [calendar / contacts / photos], which Thomas granted. In the following, you can see a screenshot of his [calendar / contacts / photos]. Have a closer look at his [calendar / contacts / photos].

In the background, the application is sending the information stored in the [calendar / contacts / photos] to the server of the app maker.

Similar text-based scenarios have been used in the security and privacy context (e.g., [24, 80]). When developing our study protocol and, more specifically, the scenario, we drew on the best practices provided by Aguinis and Bradley [5]. We drew inspiration from Harbach et al. [26] to create the above scenario illustrating various types of data on a typical smartphone: calendar, contacts, and photos. These permissions cover varying levels of privacy concerns as people have reported high privacy concerns regarding photos, medium pertaining to contacts, and low concerns about calendar [6]. Further, the three permissions we selected pertain to data explicitly entered/created by users themselves [18], rather than controller-inferred data (e.g., likes or tastes) or automaticallycollected data (e.g., geographical coordinates). Calendar, contact, and photo information is directly under the control of each user in terms of adding it to the device as well as sharing it with apps. Moreover, for most users, all three of these permissions typically involve the information of other people.

People's decisions regarding app adoption and subsequent information disclosure are influenced by a variety of contextual considerations $[43,82]$. For instance, before granting a permission that can expose the data of their friends, users may consider whether the requested information is useful for the functionality of an SNS app [57]. As we wanted to avoid these influences having a differential effect across conditions and affecting our comparative analyses, we chose a generic framing for the purpose of the app. Previous work (e.g., [55-57]) used similar generic scenarios that lack specific app information (e.g., name, purpose). We similarly did not explicitly specify whether the requested permission was mandatory for the use of the fictional app because permissions dialogs do not mention this information. While context (e.g., prior knowledge, app purpose) may influence perceptions regarding this matter, our study involves cross-condition comparisons that are unaffected by such operational detail and context due to random assignment.

The Thomas persona helped avoid the impact of individual variations in private information present on the devices of the participants. We emphasized that participants should answer "assuming they were Thomas." Moreover, random assignment to conditions means that cross-condition comparisons are unaffected by the use of a persona-based vignette as well as by the extent to which the Thomas persona matched the participants. Further, presenting the vignette minimized the possibility of biases based on a person's past decisions regarding app permissions [24, 25]. 
Table 1. Information presented in the visual depictions in each study condition. The corresponding visual depictions are in Appendix C.

\begin{tabular}{lcc}
\hline & Non-persons & Persons \\
\hline Calendar & Classes & Personal events \\
Contacts & Companies & People \\
Photos & Cities and landscapes & Parties, trips, etc. \\
\hline
\end{tabular}

For each of the three permissions mentioned in the scenario, we prepared corresponding visuals depicting information that either contained nothing regarding other people (Non-persons) or included other persons (Persons). Compared to text, images are more easily processed and elicit greater emotional response [30, 34, 37, 38]. Moreover, images are typically remembered better than words $[19,76]$. As such, a visual presentation of the information was intended to create a more vivid and memorable impression that lasted while answering questions relevant to the scenario during the short study. In contrast to Weidman et al. [80], who used third-person scenarios as we did in our study, our vignettes increase the level of immersion and realism via visual stimuli as recommended by Aguinis and Bradley [5]. Heslin et al. [28] argue that greater immersion can enhance external validity since it facilitates a stronger connection to the essential features of the real world.

Table 1 describes the information presented in the visual depictions for each of the six study conditions. The corresponding visual depictions are provided in Appendix C. Note that our operationalization of 'Non-persons' and 'Persons' is based on whether the data contains any information about other people. While it is possible that information without people is considered private by the individual, this factor typically has no bearing on the privacy of others, i.e., interdependent privacy, which is the aspect investigated in our study. As a result, individual privacy concerns pertaining to calendar, contacts, and photos in the Non-persons operationalization as described above do not impact our data and findings.

$\mathrm{Pu}$ and Grossklags [57] used a conjoint study for SNS apps, where participants chose between app versions. To avoid the high cognitive burden of a conjoint study (due to the need to compare many factors and app versions), we instead used a between-subjects factorial (i.e., Non-persons vs. Persons; different permissions) vignette with a single stimulus per condition (i.e., a visual presentation of data on Thomas's phone).

All three authors of the study worked together on developing and iteratively refining the scenario description to ensure that it accurately communicated the intended message and permitted the systematic experimental manipulation we desired. Further, we piloted the study by distributing it among a few students and postdoctoral scholars who are native speakers of English. The pilot testers confirmed the clarity and comprehension of the scenario. ${ }^{1}$

\subsection{Study Procedure and Deployment}

Upon reading information about the study and indicating their consent to participate, participants were randomly assigned to one of the six study conditions. We then presented them with the vignette assigned to their study condition, followed by the visual depiction of the information corresponding to the vignette.

\footnotetext{
${ }^{1}$ We received similar affirmation of clarity and comprehension from several participants in the form of open-ended comments praising us for the ease and clarity of the study. There were no comments mentioning any issues or containing complaints.
} 
We included two manipulation checks to ensure that participants understood the presented scenario. First, we asked a question to determine whether participants perceived the provided images to contain data of other people. This manipulation check verified that participants understood the type of information (i.e., Non-persons or Persons according to the operationalization described above) that they were shown (see Manipulation Check 1 in Appendix B). Second, we asked participants to confirm the permission covered by the scenario. For both manipulations checks, if participants chose the option that did not match the presented scenario, we flagged the incorrect answer and showed them the correct answer. Taken together, the two manipulation checks reinforced our intended priming delivered via the image presented for the respective study condition (e.g., Figure C.3 lists contacts that are mostly organizations [Non-persons condition for Contacts], and Figure C. 4 shows a contact list containing mostly people [Persons condition for Contacts]) Our operationalization of the manipulation checks is in line with the findings of Oppenheimer et al. [45], indicating that forcing inattentive participants to read instructional material thoroughly and pass associated tests "homogenizes" the sample and increases the signal-to-noise ratio, thus providing greater statistical power. In contrast, removing participants who fail manipulation checks poses the risk of "serious bias in estimation, as dropping subjects may induce an asymmetry across treatment arms" [10].

After participants cleared the two manipulation checks as described above, we asked them whether installing the app in the scenario would jeopardize the privacy of Thomas and his friends. Next, we asked whether the responsibility for protecting the information of Thomas's friends lay with Thomas or the app developer. We then instructed participants to put themselves in Thomas's position and decide whether to install the app described in the scenario. We further asked how much participants would be willing to pay for a version of the app that offered the same functionality without requiring the permission requested in the scenario. In the subsequent sections of the study, we asked questions taken from $\mathrm{Pu}$ and Grossklags [57] aimed at measuring the following constructs: privacy knowledge, other-regarding privacy, own privacy concerns, and privacy concerns of friends. We concluded the study by collecting basic demographic information. We embedded an attention check question within the study to detect non-attentive participants [16].

We deployed the study on AMT during June 2019. We restricted participation to those who resided in the United States and had completed more than 100 AMT tasks with an approval rating of $95 \%$ or higher. The study task included a link to the online questionnaire. At the end of the study, we provided participants with a randomly-generated unique code to be entered on AMT to receive compensation for completing the study. All those who entered the correct code received US $\$ 1$, even if they failed the attention check. With an average study completion time of about 6 minutes, the compensation translates to $\$ 10 /$ hour.

\subsection{Sample}

Of the 665 participants who completed the study and entered a completion code on AMT, we excluded thirteen who failed the attention check, eight who completed the study too quickly to be able to answer meaningfully (more than two standard deviations less than the median completion time), and eleven who completed the study twice. The remaining 633 participants were distributed roughly equally among the six study conditions (Calendar-Non-persons: 103, Calendar-Persons: 106, Contacts-Non-persons: 105, Contacts-Persons: 107, Photo-Non-persons: 107, and Photo-Persons: 105). The sample contained slightly more men $(57 \% ; n=363)$ than women $(42 \% ; n=263)$, with two $(0.32 \%)$ preferring not to answer and five $(0.79 \%)$ describing themselves as agender, genderfluid, or non-binary. Most participants were under 44 years of age with the greatest proportion $(48 \% ; n=305)$ in the $25-34$ age range and a quarter $(25 \% ; n=159) 35-44$ years old. Only nine participants $(1.4 \%)$ 
reported being older than 64 . Nearly half $(48 \% ; n=301)$ of the participants held an undergraduate degree; nearly a third $(32 \% ; n=203)$ indicated attending some college but without a degree; $59(9.3 \%)$ reported completing a Master's degree; and twelve (1.9\%) mentioned holding a Doctoral degree. The majority of the participants $(52 \% ; n=332)$ earned between US $\$ 25,000$ and US $\$ 75,000$ annually. About half $(50 \% ; n=315)$ of the participants were single and never married, and more than half $55 \%(n=347)$ reported having no children. Almost all participants $(96 \% ; n=610)$ indicated living in the United States for six years or longer. Participants were split roughly 2:1 in terms of Android $(69 \% ; n=435)$ and iOS $(31 \% ; n=197)$ usage, respectively. The distribution of these demographic characteristics of the sample was roughly equal across each of the six study conditions.

\subsection{Data Analysis}

We used Shapiro-Wilk tests to test whether the variables of interest were normally distributed. Whenever the assumption of normality was not violated, we used ANOVA to compare across study conditions. When variables were not normally distributed, we checked for differences across conditions using the non-parametric Mann-Whitney or Kolmogorov-Smirnov tests in pairwise fashion, with Bonferroni corrections applied to the $p$-values prior to determining statistical significance. We used the standard technique for applying the Bonferroni correction such that the original $p$-value was multiplied by $N$, where $N$ is the number of tests conducted pertaining to a given hypothesis. We used pairwise non-parametric tests with Bonferroni corrections instead of Kruskal-Wallis tests because we specifically wanted to examine which pairwise differences were statistically significant [17].

Further, we examined the text responses to the only open-ended question in the questionnaire: "Is there anything else you would like to tell us?" Two of the authors independently filtered out responses that were blank or mere expressions of gratitude in order to retain only those comments that were connected to the content of the study. There was $100 \%$ agreement between the two coders, with both flagging the same 33 comments as warranting examination (see Appendix A). Given the relatively small number of useful comments, we could perform little in-depth analysis/coding. However, the comments do include quotes that provide illustrative support when discussing the findings.

\subsection{Limitations}

Before proceeding to the findings, we point out a few important limitations of the method. We rely on self-reporting in regard to a fictitious scenario that held no real-world consequences for participants. Moreover, the scenario purposely excluded cues, such as ratings, reviews, developer information, description, purpose, contextual detail, etc., that people tend to use when making app-related decisions. Note that random assignment to study conditions distributes the impact of these factors equally across all study conditions, thereby having no effect on the conclusions drawn by comparing across conditions as we do in this paper. However, further research is needed to verify the extent to which the findings based on self-reports generalize to real-world behavior. To that end, future work could compare responses obtained by asking people about data on their own devices.

We assume that information that does not contain people has no bearing on the privacy of others. While this is certainly true for the conditions used in our study, it should be kept in mind that interdependent privacy concerns may not always be so clear-cut in reality because privacy concerns vary across people and contexts [70]. For example, depending on circumstances, a person sharing a photo of another person's car could be deemed by the other party as a violation of privacy even if no people are present in the photo. 
This first exploration is limited to three prominent permissions (i.e., calendar, contacts, and photos) that have a high likelihood of involving the information of other people. Future work should study additional permissions, such as microphone, that could gather data of other users. Although our study was designed to compare across study conditions, it might be useful to establish a baseline for absolute values for privacy concern levels for self and others by collecting data without any priming.

Finally, the sample is drawn from a specific platform and composed of residents of the United States. Verifying generalizability would require studies with samples from other cultures.

\section{FINDINGS}

We analyzed participant responses to answer the three research questions presented in Section 1. Prior to conducting analyses specific to each research question, we checked whether any differences across conditions could be associated with differences in underlying privacy knowledge and concern of the participants in each condition. We used ANOVA for the corresponding constructs: privacy knowledge, other-regarding privacy, own privacy concerns, and privacy concerns of friends. We found no statistically significant differences across conditions for these aspects. Similarly, ANOVA found no statistically significant differences across conditions in terms of participant views regarding potential for privacy violation and comfort with granting access for any of the three permissions: calendar, contacts, and photos.

We next computed the following constructs based on averaging the answers to the corresponding questionnaire items: willingness to install, thomas privacy concern, and thomas friends privacy concern, indicating the willingness to install the app, concern for Thomas's privacy, and concern for the privacy of Thomas's friends, respectively. Recall that the questionnaire asked participants to imagine themselves as Thomas, thus the latter two constructs serve as proxies for corresponding privacy concerns for self and others, respectively. We used these constructs to analyze differences across study conditions pertaining to our research questions. We discuss each in turn.

\subsection{Privacy of Others (RQ1)}

After examining the responses regarding Thomas's privacy, we investigated participant responses pertaining to the privacy of Thomas's friends separately for the Non-persons and Persons treatment conditions. In both cases, ANOVA found statistically significant differences (Non-persons conditions: $F=8.66, p<0.001$; Persons conditions: $F=10.30, p<0.001$ ). Participants expressed statistically significantly lower privacy concerns for Thomas's friends (mean 2.80 vs. 3.64 for Thomas in the Non-persons conditions and mean 3.82 vs. 3.98 in the Persons conditions). Yet, the difference in the means is more marginal in the Persons conditions compared to the substantially larger difference in the Non-persons conditions.

Since Shapiro-Wilk tests showed that the privacy concern for Thomas's friends was not normally distributed in any of the conditions, we employed non-parametric pairwise Mann-Whitney tests with Bonferroni corrections to examine how the differences were impacted by the permission type. The results echoed those found for privacy concern for Thomas, with the concern for the privacy of Thomas's friends being statistically significantly lower for the calendar permission compared with contacts and photos (see Figures 1 and 2):

- Non-persons conditions: Mean - Calendar: 2.40, Contacts: 3.13, Photos: 2.87; $W=3716$ for calendar vs. contacts, $p<0.001 ; W=4387$ for calendar vs. photos, $p<0.05$; 


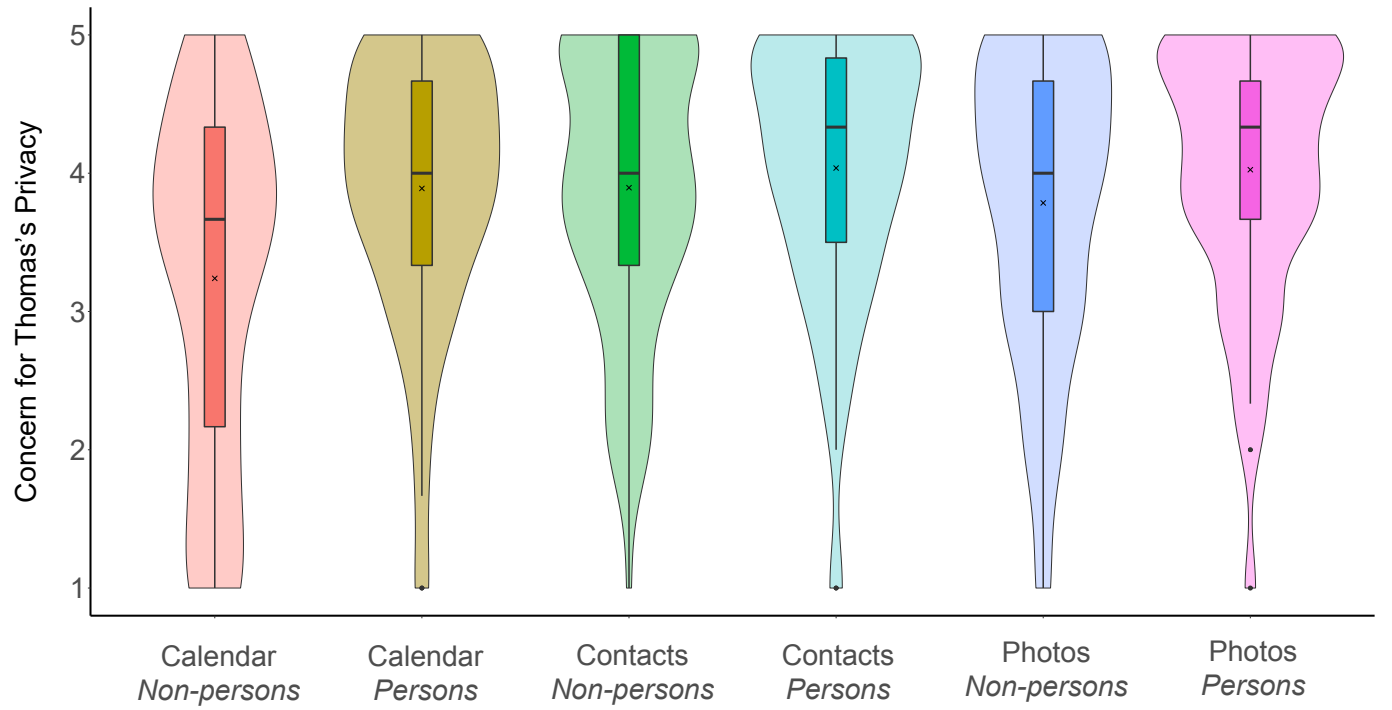

Fig. 1. Violin plots showing concern for Thomas's privacy for the Non-persons and Persons study conditions for each of the three permissions: Calendar (cal), Contacts (con), Photos (pho) on a 5-point scale, with higher values indicating higher privacy concern.

- Persons conditions: Mean - Calendar: 3.46, Contacts: 4.07, Photos: 3.93; $W=3759$ for calendar vs. contacts, $p<0.001 ; W=4178$ for calendar vs. photos, $p<0.05$

For the Non-persons as well as Persons conditions, we found no statistical differences in the concern expressed for Thomas's friends based on age, gender, or marital status.

Further, ANOVA indicated no statistical differences for participant views regarding whether Thomas or third parties were responsible for protecting the data of Thomas's friends. However, Kolmogorov-Smirnov tests with Bonferroni corrections found that participants in the Persons conditions put slightly higher onus on Thomas $($ mean $=4.15)$ than on third parties $($ mean $=3.97)$ for protecting the data of Thomas's friends $(D=0.1258, p<0.05)$. Although the means follow the same pattern in the Non-persons conditions (Thomas $=3.98$ vs. Third Parties $=3.83$ ), the difference is not statistically significant.

\subsection{Interdependent Privacy Priming (RQ2)}

The analyses above already uncovered several differences between the Non-persons and Persons conditions. We delved deeper into this aspect by comparing the Non-persons and Persons conditions for each of the three permissions (see Table 2, and Figures 1 and 2). As Table 2 and Figures 1 and 2 show, making the impact on others vividly visible in the Persons conditions resulted in notable increases in participant concerns for the privacy of Thomas's friends, with the differences for all three permissions compared to the corresponding Non-persons conditions being statistically significant at the 0.001 level based on Mann-Whitney tests with Bonferroni corrections. We found the statistical differences to apply regardless of age, gender, or marital status.

Notably, ANOVA revealed that the differences in the intent for installing the app found in the Non-persons conditions (see subsection 4.3) were absent in the Persons study conditions; we found 
Table 2. Means for Privacy Concern for Thomas (top) and Thomas's Friends (bottom) across study conditions on a 5 -point scale, with higher values indicating higher privacy concern.

\begin{tabular}{lccc}
\hline & \multicolumn{3}{c}{ Thomas's Privacy (Self) } \\
& Non-persons & Persons & Difference \\
\hline Calendar & 3.24 & 3.89 & $0.65^{* * *}$ \\
Contacts & 3.90 & 4.04 & 0.14 \\
Photos & 3.79 & 4.03 & 0.24 \\
\hline \multicolumn{4}{c}{} \\
& \multicolumn{3}{c}{ Thomas's Friends' Privacy $($ Others) } \\
& Non-persons & Persons & Difference \\
\hline Calendar & 2.40 & 3.46 & $1.06^{* * *}$ \\
Contacts & 3.13 & 4.07 & $0.94^{* * *}$ \\
Photos & 2.87 & 3.93 & $1.06^{* * *}$ \\
\hline \multicolumn{4}{c}{ Statistical significance level: } \\
\end{tabular}

Table 3. Median and mean amounts in US Dollars that participants were willing to pay to prevent data access.

\begin{tabular}{lcccc}
\hline & \multicolumn{2}{c}{ Median } & \multicolumn{2}{c}{ Mean } \\
& Non-persons & Persons & Non-persons & Persons \\
\hline Calendar & $\$ 2.00$ & $\$ 3.00$ & $\$ 9.40$ & $\$ 17.38$ \\
Contacts & $\$ 2.00$ & $\$ 2.00$ & $\$ 4.82$ & $\$ 10.32$ \\
Photos & $\$ 2.00$ & $\$ 3.00$ & $\$ 8.21$ & $\$ 10.33$ \\
\hline
\end{tabular}

no statistical differences between permission types in this regard (Means - Calendar: 2.59, Contacts: 2.21, Photos: 2.39; Medians - Calendar: 3.00, Contacts: 2.00, Photos: $2.00 ; F=2.37, p=0.09$ ). In comparison to the corresponding Non-persons conditions, the installation intent decreased for calendar (3.09 vs. $2.59 ; W=6642, p<0.05$ ), thus bringing it in alignment with the low intents for contacts and photos (which did not differ statistically across the Non-persons and Persons conditions).

In addition, surfacing other persons in the shown visual information served to raise the concern for Thomas's privacy for the calendar permission as well and brought it in alignment with the concerns for contacts and photo permissions, which were already much higher (see Section 4.3) Pairwise Mann-Whitney tests with Bonferroni corrections showed that the raised concerns for the calendar permission could be attributed to males (Mean - Non-persons: 3.09, Persons: 3.92; Median - Non-persons: 3.33, Persons: 4.00; $W=1149, p<0.001$ ) and those under 35 (Mean - Non-persons: 3.03, Persons: 3.81; Median - Non-persons: 3.33, Persons: 4.00; $W=1220, p<0.01$ ). The impact of age may have been due to our privacy priming for the calendar condition being based on university classes, which are typically more relevant for the younger population.

In all study conditions, we asked participants to provide the maximum monetary amount they would be willing to spend for a version of the app that would provide the same functionality without accessing calendar, contacts, or photos, respectively. For all three permissions, we found that participants in the Persons conditions were willing to pay more than those in the corresponding Non-persons conditions (see Table 3). 


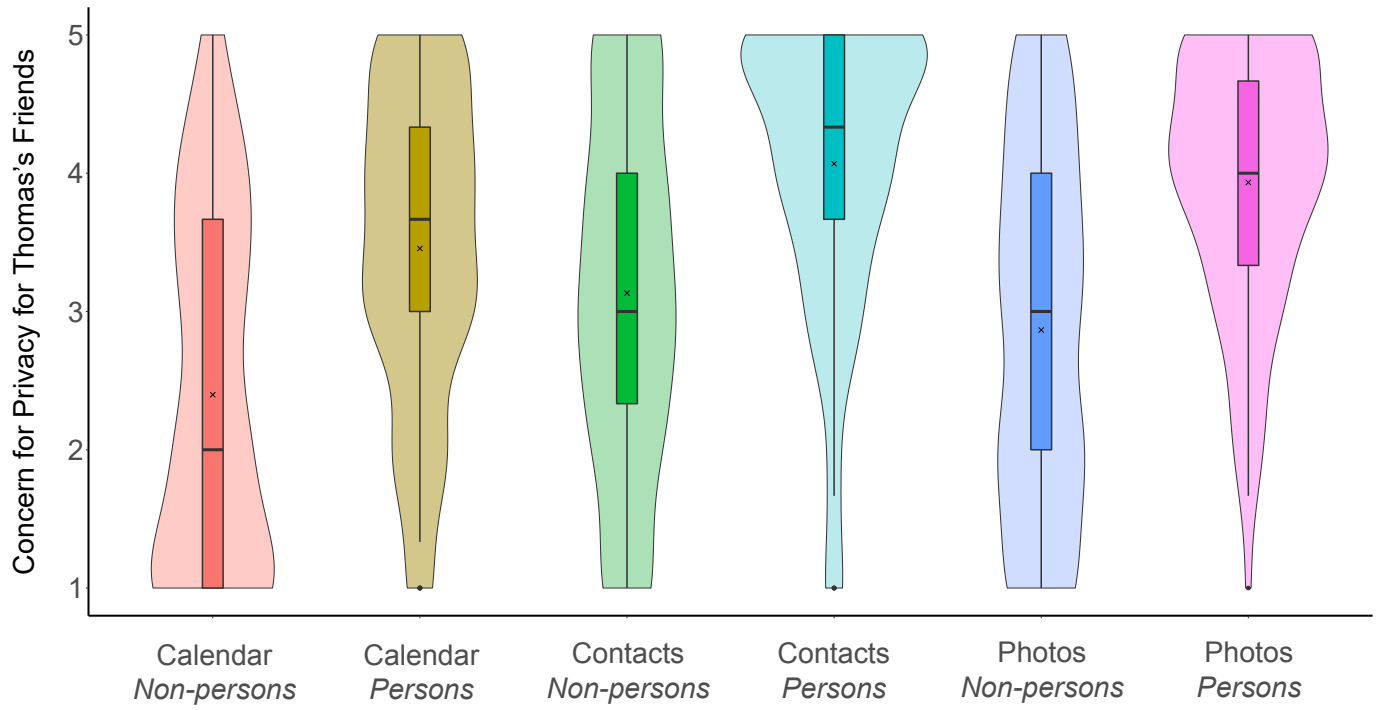

Fig. 2. Violin plots showing concern for privacy for Thomas's friends for the Non-persons and Persons study conditions for each of the three permissions: Calendar (cal), Contacts (con), Photos (pho) on a 5-point scale, with higher values indicating higher privacy concern.

\subsection{Permission Type (RQ3)}

We compared the concern for Thomas's privacy for each of the three permission types separately for the Non-persons and Persons conditions. We found that the privacy concerns for Thomas showed statistically significant differences across the three permission types, but only for the Non-persons conditions $(F=10.44, p<0.001)$. This indicates that people's baseline privacy judgments differ based on the type of permission, i.e., certain permissions are deemed inherently more privacysensitive than others. However, these differences may be nullified when people are primed with privacy-evoking information. In the rest of this subsection, we limit the discussion to the three Non-persons conditions.

We further analyzed the Non-persons conditions using pairwise comparisons. A Shapiro-Wilk test for normality showed that concern for Thomas's privacy violated the assumption of normality. Therefore, we used non-parametric Mann-Whitney tests with Bonferroni corrections, finding that the concern for Thomas's privacy was statistically significantly higher for contacts (mean $=3.90$, median $=4.00)$ and photos $($ mean $=3.79$, median $=4.00)$ than for calendar $($ mean $=3.24$, median $=$ 3.67) with $W=3793.5(p<0.001)$ and $W=4113(p=0.004)$, respectively. There was no statistical difference between contacts and photos.

We obtained similar results when examining participant ratings for privacy-sensitivity and comfort for granting these permissions to smartphone apps. Since Shapiro-Wilk tests for normality showed that these variables violated the assumption of normality, we used pairwise non-parametric Kolmogorov-Smirnov tests with Bonferroni corrections (see Table 4). The tests showed that participants considered it more privacy sensitive to grant access to contacts and photos than to the calendar $(p<0.04)$, and they were less comfortable doing so $(p<0.05)$. Similarly, ANOVA revealed 
Table 4. Privacy Sensitivity and Comfort by Permission Type in the Non-persons Study Conditions $(n=315)$ on 5-point scales, with higher values indicating higher privacy sensitivity and higher access comfort.

\begin{tabular}{lcccc}
\hline & \multicolumn{2}{c}{ Privacy Sensitivity } & \multicolumn{2}{c}{ Access Comfort } \\
& Mean & Median & Mean & Median \\
\hline Calendar & 3.92 & 4.00 & 2.77 & 3.00 \\
Contacts & 4.28 & 5.00 & 2.17 & 2.00 \\
Photos & 4.26 & 5.00 & 2.39 & 2.00 \\
\hline
\end{tabular}

statistically significant differences in the intent for installing the app $(F=16.28, p<0.001)$. Pairwise Mann-Whitney tests with Bonferroni corrections showed that the means for installation intent (on a 1-5 scale) were statistically significantly different $(p<0.01)$ for each of the three permissions (Calendar: 3.09, Contacts: 2.10, Photos: 2.61).

Within each permission type, we found no statistically significant differences based on age, gender, or marital status, except for the influence of age on contacts. A Bonferroni-corrected Mann-Whitney test showed that those older than 35 reported higher privacy concern for Thomas compared to that reported by the younger participants (mean $=4.23$ vs. $3.68 ; W=1558 ; p<0.01$ ).

\subsection{Demographics}

As described in the above subsections, most of our results comparing between study conditions apply independent of demographics, with a few exceptions. Similarly, we found no differences between Android and iOS users.

However, when examining demographic influences applicable across all conditions in general, we found effects in line with the literature. Specifically, Mann-Whitney tests showed that concern for privacy when installing apps was slightly higher for older participants compared to those younger than 35 (mean: 4.33 vs. 4.03 ; median: 5.00 vs. $4.00 ; W=57175, p<0.001$ ) and for females compared to males (mean: 4.28 vs. 4.07 ; median: 5.00 vs. $4.00 ; W=41769, p<0.01$ ). The latter could be partially attributed to males exhibiting greater confidence in their ability to control sharing of personal information with app developers (mean: 3.04 vs. 2.63; median: 3.00 in both cases; $W=56400, p<0.001$ ).

There were no notable gender differences in the number of apps, contacts, and private or nonprivate calendar events reported to be present on the phone. However, females reported having substantially more photos on their phones compared to males (mean: 1468 vs. $1067 ; W=39016$, $p<0.001)$. Yet, there were no statistically significant gender differences when comparing privacy concerns across study conditions, even for the conditions involving the photo permission.

Interestingly, the above baseline differences based on demographics did not translate into differences in the money that participants were willing to pay for an app version that did not access personal information. The only exception was unmarried participants who were willing to pay less than the rest for preventing access to private calendar events (mean: $\$ 3.45$ vs. $\$ 34.21$; median $\$ 2.00$ vs. $\$ 5.00 ; W=970.5, p<0.05)$.

\section{DISCUSSION}

In summary, we found that smartphone users show lower regard for the privacy of others compared to concerns for their own privacy, regardless of the specific permission in question. At the same time, we found that the magnitude of the discrepancy can be reduced by priming people with person-based visual depictions prior to making decisions that can affect the privacy of others. However, our results suggest that the mitigating effect of the priming is dependent on the type of 
permission in question and may depend on the baseline level of privacy concern for that permission. On a related but minor note, we discovered that people might be willing to pay a small premium for app versions that are more protective of other people's privacy. We found that these results show no notable influence of smartphone platform or demographics such as age, gender, and marital status.

The findings related to differences between privacy concerns for self and others affirm and extend prior work on interdependent privacy in the SNS context [57] to the context of smartphone app permissions. Specifically, we confirmed that, just like SNS, people tend to care more about the implications of their actions for their own privacy rather than the privacy of others who might be affected by those actions. As a participant mentioned in open-ended comments: "I have never thought about the privacy of my friends."

Interestingly, our findings suggest that this focus on the self is not necessarily due to shirking responsibility toward protecting the privacy of others. In fact, in all study conditions, participants indicated that Thomas was as responsible for protecting the privacy of other people's data on his device as third parties, such as app developers. We conjecture that people's lower concern for the privacy of others might arise from the matter not being prominent enough in their minds when making decisions that could potentially affect others. That said, an alternative explanation could be that participants were instead expressing that the decision to grant permission to the app comes with the responsibility of protecting the privacy of others potentially affected by the consequences of that decision.

Indeed, in the Persons study conditions, when primed with visual depictions that involved other people, the concern for the privacy of others increased substantially for all three permissions we studied. Similar to the literature on nudging people to consider their own privacy (e.g. [2, 7, 79]), our findings show that priming can help elevate the attention given to the privacy of others as well. As a participant remarked: "This study definitely made me more aware since I do have photos of my family, as well as other photos, too. I did not think I had anywhere near that many photos in my phone."

However, recent experimental findings suggest that explicitly priming individuals for the "privacy of the person" (in a photo) may lead to paradoxical outcomes [8]. In particular, if people feel that privacy of the person in the photo is not likely to be violated, they might be more willing to share such content in comparison to a more neutral scenario. This suggests that there might be differences between visual and textual priming as well as between priming that explicitly mentions privacy and that which does not. Alternatively, it might be the case that people might be concerned about the privacy of others (as they reported in our study) and choose to share the information regardless (as reported by Amon et al. [8]). Further research is needed to unpack these aspects.

We further found that the impact of priming for one's own privacy is the most beneficial for permissions where the baseline concern is lower. This is to be expected owing to the ceiling effect; when baseline concern is already close to the highest, there is little room for further movement While nudging has generally been explored for the most privacy-sensitive matters, our findings suggest that it might be even more necessary in cases where people are more likely to be cavalier about privacy and have their guards down as a consequence.

We uncovered that privacy concerns - for self or others - can vary depending on the permission being sought by an app. Among the permissions we studied, access to the calendar evoked the least concern. The comparatively lower concern could potentially be explained by lower adoption of calendar-related apps and/or lower amounts of sensitive data present in calendar entries. Somewhat surprisingly, participants expressed slightly lower privacy concerns about photos than contacts, despite having 100 s or 1000 s of photos on their devices. We imagine that greater familiarity with apps that use photos and/or the device camera as well as the static nature of contact information 
may be a factor behind these findings. Further research is needed to understand how privacyrelated decision making is influenced by people's relative privacy rankings for various smartphone permissions.

When considering our findings regarding payment amounts for a more private version of the app, it should be kept in mind that we asked participants to answer by imagining themselves as Thomas. Therefore, the valuations reflect judgments for Thomas rather than participants' own willingness to pay. That said, the data can still serve as indirect indication for people's willingness to pay as the fictitious nature of Thomas means that the answers must necessarily be based upon relating to the typical experiences of one's own life or the lives of those one knows who might be similar to Thomas.

Finally, we found that people's answers to questions covering various aspects of privacy concerns clustered toward the high ends of the scale. This is similar to findings in the SNS context. For example, Krasnova et al. [35] report that concerns regarding photo permissions are invariably high irrespective of whether one considers such data about oneself $(4.59 / 5.0)$ or others $(4.50 / 5.0)$ Moreover, most $(82 \%, n=517)$ of the participants in our study were aware that granting a permission allows an app to access all of the data associated with that permission and transfer it out of the device. When considering the general tendency of people not to scrutinize the dialogs for permission requests $[22,33,40]$, the responses of our participants serve as yet another illustration of the privacy paradox [42] and/or privacy resignation [29, 62, 83]. This is reflected in participant comments such as: "I don't like it when apps require such access, but oftentimes I go ahead and download them anyways." Additionally, the responses may also be indicative of the growing awareness and importance of technology-related privacy issues among the general population. As a result, our findings point to the increasing methodological limitations of asking people to self-report privacy concerns as their utility for performing privacy segmentation may be significantly reduced owing to the low variance in the answers.

\section{IMPLICATIONS}

Our findings suggest a number of avenues for user experience (UX) and system improvements that can help people consider interdependent privacy and avoid multi-party privacy conflicts.

Smartphone operating systems and apps have recognized that certain permissions are privacyand security-sensitive and need to be handled with extra care. For instance, the Android operating system classifies permissions into normal and dangerous based on privacy and security implications $[1,65]$. However, the classification is geared mostly toward developers, not users. Our findings call for refinements to the user interface to take into account the differences in privacy properties of different permissions. The high-sensitivity location permission has typically received the most research and public policy attention (e.g., [7, 47, 48, 61, 84]). Our findings indicate that other permissions that raise privacy concerns could benefit from further research and permission-specific public policy guidance. Such research could facilitate a rating/ranking of permissions and apps based on privacy sensitivity. For example, Facebook's app permission system distinguishes between permissions pertaining to the data provided by users themselves and that contributed by others. Yet, the options presented during app installation largely omit tangible options for the user, a point addressed in previous design [78] and experimental [77] studies.

In this regard, solutions could build upon the UX of the prominent warning displayed when an app is actively using location information. For instance, similar to location [7], alerts and notifications can be explored for drawing people's attention to app access to other types of data on their devices. Moreover, permission interfaces could be made customizable to allow users to arrange permissions based on sensitivity. 
Additionally, the permissions model for smartphone operating systems could be enhanced by enabling more granular control over data access. However, there is a trade-off between the increased control provided by greater granularity and the greater burden and potential confusion in managing the settings. The specifics of this trade-off depend on the context (i.e., some permissions may be more suitable for greater granular control than others) as well as the individual (i.e., some users may prefer greater control, despite the burden). Empirical investigation is needed to understand how to implement these controls to achieve optimal trade-offs across contexts and users.

Already existing mechanisms could be extended to take into account considerations for interdependent privacy in addition to the user's own privacy. The latest version of iOS, for instance, includes mechanisms whereby users can choose to share only a subset of their photo library by explicitly selecting photos that an app is permitted to access. Related enhancements could conversely permit users to protect specific pieces of information from being accessed by applications. For example, users could choose to permit apps to access photos except those that contain other people. Alternatively, or in addition, users could be allowed to create app-specific sandboxes within specific device-wide data repositories, such as photos.

While manual specification settings for thousands of pieces of information is surely impractical and burdensome, quick mechanisms for flagging sensitivity could help mark the most privacysensitive items, which might be much fewer compared to the size of the whole set. Moreover, classifiers based on machine learning [64] and/or computer vision [66, 74] could help automate the marking task. For instance, such automation could enable users to indicate that photos in which faces of other people are detected should not be shared without explicit permission. Olteanu et al. [44] have demonstrated that the process of seeking such consent from others could be automated and integrated within existing platforms. Additionally, being forced to seek consent could serve as a 'precautionary' mechanism against sharing other people's information [14].

In terms of helping protect the privacy of others, current mechanisms and designs for privacy nudges can be enhanced by drawing attention to the likely impact on others. For instance, prior to making decisions that can impact the privacy of other parties, relevant snippets of the underlying information that include the other parties can be surfaced. For example, the dialog box that asks for the photo permission can show a photo strip with a few photos of other individuals instead of selfies or photos without any people. Such nudges can counter people's tendency to underestimate the amount and sensitivity of the information stored on their devices [15]. In the context of social networking sites, Cherubini et al. [14] found that such 'dissuasive' mechanisms are particularly effective for frequent sharers and older users. Makers of smartphone operating systems could similarly explore priming, alerts, and notifications for app access to others' data and improve the granularity of permissions models (which iOS now does, but only for photos). Permissions dialogs could additionally highlight whether data of others is transferred to a third party. While these suggestions are a useful starting point, we encourage a broad exploration of the design space of nudges to identify those that are effective and convenient for facilitating consideration of the privacy of others.

Additionally, the architecture of the system and corresponding access permissions should be augmented to allow users to specify how their data stored on others' devices should be handled. Such functionalities could mimic and extend the current mechanisms related to photo tagging on SNS where users are able to specify that tags by others may not be shown publicly and delete tags when others tag them in photos. For instance, such architectures could permit Alice to specify that she would prefer that her contact information stored on Bob's phone not be shared with an app even if Bob grants the app permission to access his contacts. Bob's contact list can surface Alice's preferences such that Bob and/or apps may make an informed decision that respects Alice's wishes. 
To hone in on the problem of interdependent privacy on mobile devices, we call for reports derived from longitudinal measurement studies. We believe that suitable data about app practices has already been collected in various projects (e.g., [36, 59]), but not yet examined from the vantage point of interdependent privacy. We further suggest that it would be valuable to seek empirical insight to understand how user rights (e.g., the right to data portability [72]) granted by privacy laws (e.g., GDPR) can be applied to address the problem interdependent privacy.

Although not central to our investigation, our findings demonstrate that willingness to pay can be affected by app permissions. The results complement studies reporting what individuals would pay for their own privacy. We did not explicitly ask why participants chose the amounts they were willing to pay, and our findings cannot quantify exact amounts. Since the only difference between the Non-persons and Persons conditions was our priming, our findings do suggest that a large enough proportion of app users might be willing to pay to protect the privacy of themselves and others whose data is present on their devices. App developers could, therefore, explore paid versions that provide privacy-preserving operation, such as reduced use of permissions, client-side processing of data, storage of data on the device (instead of the cloud), limiting the use of data of those other than the device user, etc. While the paid versions may not be adopted by everyone, they can help expand the user base to be inclusive of the more privacy-conscious individuals who would otherwise choose not to use the app at all and provide an alternate revenue stream in lieu of data monetization.

As the literature highlights (see Section 2), decisions over "shared content" are difficult, particularly after it has been already made available to others. Therefore, it is better to intervene early by highlighting the privacy risk to others because of one's decision to share one's data. Marketplaces and regulators could scrutinize apps for harvesting data of other parties since this multiplies the effect of data harvesting and imposes negative externalities on others.

\section{CONCLUSION}

Mobile devices increasingly store privacy-sensitive data of other parties with whom the user maintains personal or professional relationships. As a result, user decisions pertaining to app permissions hold consequences for privacy beyond simply the individual privacy of the user. Our findings show that people often overlook privacy of others but can be reminded to take it into account by appropriate priming when making privacy-relevant decisions. We call for nudges, permissions interfaces, and system architectures to be designed by approaching privacy not simply as an individual issue but as anterdependent and collective concern.

\section{ACKNOWLEDGMENTS}

We thank the participants of the study. We are grateful to the anonymous reviewers for their constructive comments. Jens Grossklags gratefully acknowledges support from the Bavarian Research Institute for Digital Transformation (bidt). Sameer Patil's involvement in the research was supported through a Visiting Professorship at the Technical University of Munich funded as part of the visiting professor program of the Bavarian State Ministry for Science and Art. The content of the paper is the work of the authors and does not necessarily reflect the views of the sponsors.

\section{REFERENCES}

[1] Yasemin Acar, Michael Backes, Sven Bugiel, Sascha Fahl, Patrick McDaniel, and Matthew Smith. 2016. SoK: Lessons Learned from Android Security Research for Appified Software Platforms. In 2016 IEEE Symposium on Security and Privacy (San Jose, CA, USA) (IEEE S\&P '16). Institute of Electrical and Electronics Engineers, 433-451. https: //doi.org/10.1109/SP.2016.33

[2] Alessandro Acquisti. 2009. Nudging Privacy: The Behavioral Economics of Personal Information. IEEE Security \& Privacy 7, 6 (2009), 82-85. https://doi.org/10.1109/MSP.2009.163 
[3] Alessandro Acquisti, Laura Brandimarte, and George Loewenstein. 2015. Privacy and Human Behavior in the Age of Information. Science 347, 6221 (2015), 509-514. https://doi.org/10.1126/science.aaa1465

[4] Alessandro Acquisti, Curtis Taylor, and Liad Wagman. 2016. The Economics of Privacy. fournal of Economic Literature 54, 2 (June 2016), 442-492. https://doi.org/10.1257/jel.54.2.442

[5] Herman Aguinis and Kyle J. Bradley. 2014. Best Practice Recommendations for Designing and Implementing Experimental Vignette Methodology Studies. Organizational Research Methods 17, 4 (2014), 351-371. https: //doi.org/10.1177/1094428114547952

[6] Mohammed M. Alani. 2017. Android Users Privacy Awareness Survey. International fournal of Interactive Mobile Technologies (ifIM) 11, 3 (2017), 130-144. https://doi.org/10.3991/ijim.v11i3.6605

[7] Hazim Almuhimedi, Florian Schaub, Norman Sadeh, Idris Adjerid, Alessandro Acquisti, Joshua Gluck, Lorrie Faith Cranor, and Yuvraj Agarwal. 2015. Your Location Has Been Shared 5,398 Times! A Field Study on Mobile App Privacy Nudging. In Proceedings of the 33rd Annual ACM Conference on Human Factors in Computing Systems (CHI '15). Association for Computing Machinery, New York, NY, USA, 787-796. https://doi.org/10.1145/2702123.2702210

[8] Mary Jean Amon, Rakibul Hasan, Kurt Hugenberg, Bennett I. Bertenthal, and Apu Kapadia. 2020. Influencing Photo Sharing Decisions on Social Media: A Case of Paradoxical Findings. In 2020 IEEE Symposium on Security and Privacy (San Francisco, CA, USA) (IEEE S\&P '20). Institute of Electrical and Electronics Engineers, 1350-1366. https://doi.org/10.1109/SP40000.2020.00006

[9] AppCensus. 2020. 1,000 Mobile Apps in Australia: A Report for the ACCC. (24 sept 2020). https: //www.acc.gov.au/system/files/1\%2C000\%20Mobile\%20Apps\%20in\%20Australia\%20\%E2\%80\%93\%20A\%20Report\% 20for\%20the\%20ACCC\%2C\%20AppCensus_0.pdf Accessed: 2021-08-22.

[10] Peter M. Aronow, Jonathon Baron, and Lauren Pinson. 2019. A Note on Dropping Experimental Subjects who Fail a Manipulation Check. Political Analysis 27, 4 (2019), 572-589. https://doi.org/10.1017/pan.2019.5

[11] Gergely Biczók and Pern Hui Chia. 2013. Interdependent Privacy: Let Me Share Your Data. In Financial Cryptography and Data Security, Ahmad-Reza Sadeghi (Ed.). Springer Berlin Heidelberg, Berlin, Heidelberg, 338-353. https: //doi.org/10.1007/978-3-642-39884-1_29

[12] danah boyd. 2012. Networked Privacy. Surveillance \& Society 10, 3 (2012), 348-350. https://doi.org/10.24908/ss.v10i3/4 4529

[13] Jin Chen, Jerry Wenjie Ping, Yunjie Xu, and Bernard C. Y. Tan. 2015. Information Privacy Concern about Peer Disclosure in Online Social Networks. IEEE Transactions on Engineering Management 62, 3 (2015), 311-324. https: //doi.org/10.1109/TEM.2015.2432117

[14] Mauro Cherubini, Kavous Salehzadeh Niksirat, Marc-Olivier Boldi, Henri Keopraseuth, Jose M. Such, and Kévin Huguenin. 2021. When Forcing Collaboration is the Most Sensible Choice: Desirability of Precautionary and Dissuasive Mechanisms to Manage Multiparty Privacy Conflicts. Proc. ACM Hum.-Comput. Interact. 5, CSCW1, Article 53 (April 2021), 36 pages. https://doi.org/10.1145/3449127

[15] Jason W. Clark, Peter Snyder, Damon McCoy, and Chris Kanich. 2015. "I Saw Images I Didn't Even Know I Had": Understanding User Perceptions of Cloud Storage Privacy. In Proceedings of the 33rd Annual ACM Conference on Human Factors in Computing Systems (CHI '15). Association for Computing Machinery, New York, NY, USA, 1641-1644. https://doi.org/10.1145/2702123.2702535

[16] Cihan Cobanoglu, Muhittin Cavusoglu, and Gozde Turktarhan. 2021. A Beginner's Guide and Best Practices for Using Crowdsourcing Platforms for Survey Research: The Case of Amazon Mechanical Turk (MTurk). Journal of Global Business Insights 6, 1 (2021), 92-97. https://doi.org/10.5038/2640-6489.6.1.1177

[17] William Jay Conover and Ronald L. Iman. 1979. On Multiple-Comparisons Procedures. Technical Report LA-7677-MS Informal Report. Los Alamos Scientific Laboratory, Los Alamos, NM, USA. https://doi.org/10.2172/6057803

[18] Paul De Hert, Vagelis Papakonstantinou, Gianclaudio Malgieri, Laurent Beslay, and Ignacio Sanchez. 2018. The Right to Data Portability in the GDPR: Towards User-centric Interoperability of Digital Services. Computer Law \& Security Review 34, 2 (2018), 193-203. https://doi.org/10.1016/j.clsr.2017.10.003

[19] Margaret Anne Defeyter, Riccardo Russo, and Pamela Louise McPartlin. 2009. The Picture Superiority Effect in Recognition Memory: A Developmental Study Using the Response Signal Procedure. Cognitive Development 24, 3 (2009), 265-273. https://doi.org/10.1016/j.cogdev.2009.05.002

[20] Mariella Dimiccoli, Juan Marín, and Edison Thomaz. 2018. Mitigating Bystander Privacy Concerns in Egocentric Activity Recognition with Deep Learning and Intentional Image Degradation. Proc. ACM Interact. Mob. Wearable Ubiquitous Technol. 1, 4, Article 132 (Jan. 2018), 18 pages. https://doi.org/10.1145/3161190

[21] Adrienne Porter Felt, Erika Chin, Steve Hanna, Dawn Song, and David Wagner. 2011. Android Permissions Demystified. In Proceedings of the 18th ACM Conference on Computer and Communications Security (Chicago, Illinois, USA) (CCS '11). Association for Computing Machinery, New York, NY, USA, 627-638. https://doi.org/10.1145/2046707.2046779

[22] Adrienne Porter Felt, Elizabeth Ha, Serge Egelman, Ariel Haney, Erika Chin, and David Wagner. 2012. Android Permissions: User Attention, Comprehension, and Behavior. In Proceedings of the Eighth Symposium on Usable Privacy 
and Security (Washington, D.C.) (SOUPS 2012). Association for Computing Machinery, New York, NY, USA, Article 3 , 14 pages. https://doi.org/10.1145/2335356.2335360

[23] Christine Geeng and Franziska Roesner. 2019. Who's in Control? Interactions in Multi-User Smart Homes. In Proceedings of the 2019 CHI Conference on Human Factors in Computing Systems. Association for Computing Machinery, New York, NY, USA, 1-13. https://doi.org/10.1145/3290605.330049

[24] James T. Graves, Alessandro Acquisti, and Ross Anderson. 2019. Perception versus Punishment in Cybercrime. The Journal of Criminal Law and Criminology 109, 2 (2019), 313-364.

[25] Jie Gu, Yunjie (Calvin) Xu, Heng Xu, Cheng Zhang, and Hong Ling. 2017. Privacy Concerns for Mobile App Download: An Elaboration Likelihood Model Perspective. Decision Support Systems 94 (2017), 19-28. https://doi.org/10.1016/j.dss. 2016.10.002

[26] Marian Harbach, Markus Hettig, Susanne Weber, and Matthew Smith. 2014. Using Personal Examples to Improve Risk Communication for Security \& Privacy Decisions. In Proceedings of the SIGCHI Conference on Human Factors in Computing Systems (Toronto, Ontario, Canada) (CHI '14). Association for Computing Machinery, New York, NY, USA, 2647-2656. https://doi.org/10.1145/2556288.2556978

[27] Rakibul Hasan, David Crandall, Mario Fritz, and Apu Kapadia. 2020. Automatically Detecting Bystanders in Photos to Reduce Privacy Risks. In 2020 IEEE Symposium on Security and Privacy (San Francisco, CA, USA) (IEEE S\&P '20). Institute of Electrical and Electronics Engineers, 318-335. https://doi.org/10.1109/SP40000.2020.00097

[28] Peter A. Heslin, Don Vandewalle, and Gary P. Latham. 2006. Keen to Help? Managers' Implicit Person Theories and Their Subsequent Employee Coaching. Personnel Psychology 59, 4 (2006), 871-902. https://doi.org/10.1111/j.17446570.2006.00057.x

[29] Christian Pieter Hoffmann, Christoph Lutz, and Giulia Ranzini. 2016. Privacy Cynicism: A New approach to the Privacy Paradox. Cyberpsychology: Journal of Psychosocial Research on Cyberspace 10, 4, Article 7 (2016). https: //doi.org/10.5817/CP2016-4-7

[30] Peter S. Houts, Cecilia C. Doak, Leonard G. Doak, and Matthew J. Loscalzo. 2006. The Role of Pictures in Improving Health Communication: A Review of Research on Attention, Comprehension, Recall, and Adherence. Patient Education and Counseling 61, 2 (2006), 173-190. https://doi.org/10.1016/j.pec.2005.05.004

[31] Mathias Humbert, Benjamin Trubert, and Kévin Huguenin. 2019. A Survey on Interdependent Privacy. ACM Comput. Surv. 52, 6, Article 122 (Oct. 2019), 40 pages. https://doi.org/10.1145/3360498

[32] Deepak Karambelkar. 2014. Spyware: A Bird's-eye View. Gulf News (13 Feb. 2014). https://gulfnews.com/technology/ consumer-electronics/spyware-a-birds-eye-view-1.1289319 Accessed: 2021-08-22.

[33] Patrick Gage Kelley, Sunny Consolvo, Lorrie Faith Cranor, Jaeyeon Jung, Norman Sadeh, and David Wetherall. 2012. A Conundrum of Permissions: Installing Applications on an Android Smartphone. In Financial Cryptography and Data Security, Jim Blyth, Sven Dietrich, and L. Jean Camp (Eds.). Springer Berlin Heidelberg, Berlin, Heidelberg, 68-79. https://doi.org/10.1007/978-3-642-34638-5_6

[34] Elizabeth A. Kensinger and Daniel L. Schacter. 2006. Processing Emotional Pictures and Words: Effects of Valence and Arousal. Cognitive, Affective, \& Behavioral Neuroscience 6, 2 (2006), 110-126. https://doi.org/10.3758/CABN.6.2.110

[35] Hanna Krasnova, Nicole Eling, Oleg Schneider, Helena Wenninger, and Thomas Widjaja. 2013. Does This App Ask for Too Much Data? The Role of Privacy Perceptions in User Behavior Towards Facebook Applications and Permission Dialogs. In Proceedings of the 21st European Conference on Information Systems (ECIS 2013 Completed Research). Article 179, 12 pages. https://aisel.aisnet.org/ecis2013_cr/179

[36] Jacob Leon Kröger, Jens Lindemann, and Dominik Herrmann. 2020. How Do App Vendors Respond to Subject Access Requests? A Longitudinal Privacy Study on iOS and Android Apps. In Proceedings of the 15th International Conference on Availability, Reliability and Security (Virtual Event, Ireland) (ARES '20). Association for Computing Machinery, New York, NY, USA, Article 10, 10 pages. https://doi.org/10.1145/3407023.3407057

[37] Annie Lang, Rachel Bailey, and Sean Ryan Connolly. 2015. Encoding Systems and Evolved Message Processing: Pictures Enable Action, Words Enable Thinking. Media and Communication 3, 1 (2015), 34-43. https://doi.org/10.17645/mac. v3i1.248

[38] Annie Lang, Robert F. Potter, and Paul D. Bolls. 1999. Something for Nothing: Is Visual Encoding Automatic? Media Psychology 1, 2 (1999), 145-163. https://doi.org/10.1207/s1532785xmep0102_4

[39] Bin Liu, Mads Schaarup Andersen, Florian Schaub, Hazim Almuhimedi, Shikun (Aerin) Zhang, Norman Sadeh, Yuvraj Agarwal, and Alessandro Acquisti. 2016. Follow My Recommendations: A Personalized Privacy Assistant for Mobile App Permissions. In Twelfth Symposium on Usable Privacy and Security (SOUPS 2016). USENIX Association, Denver, CO, 27-41. https:/www.usenix.org/conference/soups2016/technical-sessions/presentation/liu

[40] Alexios Mylonas, Anastasia Kastania, and Dimitris Gritzalis. 2013. Delegate the Smartphone User? Security Awareness in Smartphone Platforms. Computers \& Security 34 (2013), 47-66. https://doi.org/10.1016/j.cose.2012.11.004

[41] Helen Nissenbaum. 2004. Privacy as Contextual Integrity. Washington Law Review 79, 1 (2004), 119-158. https: //heinonline.org/HOL/LandingPage?handle=hein.journals/washlr79\&div=16 
[42] Patricia A. Norberg, Daniel R. Horne, and David A. Horne. 2007. The Privacy Paradox: Personal Information Disclosure Intentions versus Behaviors. Journal of Consumer Affairs 41, 1 (2007), 100-126. https://doi.org/10.1111/j.17456606.2006.00070.x

[43] Katarzyna Olejnik, Italo Dacosta, Joana Soares Machado, Kévin Huguenin, Mohammad Emtiyaz Khan, and JeanPierre Hubaux. 2017. SmarPer: Context-Aware and Automatic Runtime-Permissions for Mobile Devices. In 2017 IEEE Symposium on Security and Privacy (San Jose, CA, USA) (IEEE S\&P '20). Institute of Electrical and Electronics Engineers, 1058-1076. https://doi.org/10.1109/SP.2017.25

[44] Alexandra-Mihaela Olteanu, Kévin Huguenin, Italo Dacosta, and J-P Hubaux. 2018. Consensual and Privacy-preserving Sharing of Multi-subject and Interdependent Data. In Proceedings of the 25th Network and Distributed System Security Symposium (NDSS 2018). Internet Society, 15 pages. https://doi.org/10.14722/ndss.2018.23002

[45] Daniel M. Oppenheimer, Tom Meyvis, and Nicolas Davidenko. 2009. Instructional Manipulation Checks: Detecting Satisficing to Increase Statistical Power. Journal of Experimental Social Psychology 45, 4 (2009), 867-872. https: //doi.org/10.1016/j.jesp.2009.03.009

[46] Federica Paci, Anna Squicciarini, and Nicola Zannone. 2018. Survey on Access Control for Community-Centered Collaborative Systems. ACM Comput. Surv. 51, 1, Article 6 (Jan. 2018), 38 pages. https://doi.org/10.1145/3146025

[47] Sameer Patil, Greg Norcie, Apu Kapadia, and Adam J. Lee. 2012. Reasons, Rewards, Regrets: Privacy Considerations in Location Sharing as an Interactive Practice. In Proceedings of the Eighth Symposium on Usable Privacy and Security (Washington, D.C.) (SOUPS 2012). Association for Computing Machinery, New York, NY, USA, Article 5, 15 pages. https://doi.org/10.1145/2335356.2335363

[48] Sameer Patil, Roman Schlegel, Apu Kapadia, and Adam J. Lee. 2014. Reflection or Action? How Feedback and Control Affect Location Sharing Decisions. In Proceedings of the SIGCHI Conference on Human Factors in Computing Systems (Toronto, Ontario, Canada) (CHI '14). Association for Computing Machinery, New York, NY, USA, 101-110. https://doi.org/10.1145/2556288.2557121

[49] Sai Teja Peddinti, Igor Bilogrevic, Nina Taft, Martin Pelikan, Úlfar Erlingsson, Pauline Anthonysamy, and Giles Hogben. 2019. Reducing Permission Requests in Mobile Apps. In Proceedings of the Internet Measurement Conference (Amsterdam, Netherlands) (IMC '19). Association for Computing Machinery, New York, NY, USA, 259-266. https: //doi.org/10.1145/3355369.3355584

[50] Anthony Peruma, Jeffrey Palmerino, and Daniel E. Krutz. 2018. Investigating User Perception and Comprehension of Android Permission Models. In Proceedings of the 5th International Conference on Mobile Software Engineering and Systems (Gothenburg, Sweden) (MOBILESoft '18). Association for Computing Machinery, New York, NY, USA, 56-66. https://doi.org/10.1145/3197231.3197246

[51] Giuseppe Petracca, Ahmad-Atamli Reineh, Yuqiong Sun, Jens Grossklags, and Trent Jaeger. 2017. AWare: Preventing Abuse of Privacy-Sensitive Sensors via Operation Bindings. In 26th USENIX Security Symposium (USENIX Security '17). USENIX Association, Vancouver, BC, 379-396. https://www.usenix.org/conference/usenixsecurity17/technicalsessions/presentation/petracca

[52] Giuseppe Petracca, Yuqiong Sun, Ahmad-Atamli Reineh, Patrick McDaniel, Jens Grossklags, and Trent Jaeger. 2019. EnTrust: Regulating Sensor Access by Cooperating Programs via Delegation Graphs. In 28th USENIX Security Symposium (USENIX Security '19). USENIX Association, Santa Clara, CA, 567-584. https://www.usenix.org/conference/ usenixsecurity19/presentation/petracca

[53] Sandra Petronio. 2002. Boundaries of Privacy: Dialectics of Disclosure. State University of New York Press.

[54] Yu Pu and Jens Grossklags. 2014. An Economic Model and Simulation Results of App Adoption Decisions on Networks with Interdependent Privacy Consequences. In Decision and Game Theory for Security, Radha Poovendran and Walid Saad (Eds.). Springer International Publishing, Cham, 246-265. https://doi.org/10.1007/978-3-319-12601-2_14

[55] Yu Pu and Jens Grossklags. 2015. Using Conjoint Analysis to Investigate the Value of Interdependent Privacy in Social App Adoption Scenarios. In Proceedings of Thirty Sixth International Conference on Information Systems (Fort Worth, TX, USA) (ICIS 2015). 20 pages. https://aisel.aisnet.org/icis2015/proceedings/SecurityIS/12/

[56] Yu Pu and Jens Grossklags. 2016. Towards a Model on the Factors Influencing Social App Users' Valuation of Interdependent Privacy. Proceedings on Privacy Enhancing Technologies 2016, 2 (2016), 61-81. https://doi.org/10.1515/ popets-2016-0005

[57] Yu Pu and Jens Grossklags. 2017. Valuating Friends' Privacy: Does Anonymity of Sharing Personal Data Matter?. In Thirteenth Symposium on Usable Privacy and Security (SOUPS 2017). USENIX Association, Santa Clara, CA, 339-355. https://www.usenix.org/conference/soups2017/technical-sessions/presentation/pu

[58] Selvakumar Ramachandran, Andrea Dimitri, Maulahikmah Galinium, Muhammad Tahir, Indirajith Viji Ananth, Christian H. Schunck, and Maurizio Talamo. 2017. Understanding and Granting Android permissions: A user survey. In 2017 International Carnahan Conference on Security Technology (Madrid, Spain) (ICCST 2017). Institute of Electrical and Electronics Engineers, 1-6. https://doi.org/10.1109/CCST.2017.8167834 
[59] Irwin Reyes, Primal Wijesekera, Joel Reardon, Amit Elazari Bar On, Abbas Razaghpanah, Narseo Vallina-Rodriguez, and Serge Egelman. 2018. “Won't Somebody Think of the Children?” Examining COPPA Compliance at Scale. Proceedings on Privacy Enhancing Technologies 2018, 3 (2018), 63-83. https://doi.org/10.1515/popets-2018-0021

[60] Jordan Robertson. 2014. Google+, 'Candy Crush' Show Risk of Leakiest Apps. Bloomberg (29 Jan. 2014). https:// www.bloomberg.com/news/articles/2014-01-29/nsa-spying-on-apps-shows-perils-of-google-candy-crush- Accessed: 2021-08-22.

[61] Muhammad Safi, Abhiditya Jha, Malak Aly, Xinru Page, Sameer Patil, and Pamela Wisniewski. 2019. Will They Share? Predicting Location Sharing Behaviors of Smartphone Users through Self-Reflection on Past Privacy Behaviors. In Workshop on Usable Security (San Diego, CA, USA) (USEC 2019). 13 pages. https://doi.org/10.14722/usec.2019.23014

[62] John S. Seberger, Marissel Llavore, Nicholas Nye Wyant, Irina Shklovski, and Sameer Patil. 2021. Empowering Resignation: There's an App for That. In Proceedings of the 2021 CHI Conference on Human Factors in Computing Systems (CHI '21). Association for Computing Machinery, New York, NY, USA, Article 552, 18 pages. https://doi.org/10.1145/ 3411764.3445293

[63] Pan Shi, Heng Xu, and Yunan Chen. 2013. Using Contextual Integrity to Examine Interpersonal Information Boundary on Social Network Sites. In Proceedings of the SIGCHI Conference on Human Factors in Computing Systems (CHI '13). Association for Computing Machinery, New York, NY, USA, 35-38. https://doi.org/10.1145/2470654.2470660

[64] Daniel Smullen, Yuanyuan Feng, Shikun Aerin Zhang, and Norman Sadeh. 2020. The Best of Both Worlds: Mitigating Trade-offs Between Accuracy and User Burden in Capturing Mobile App Privacy Preferences. Proceedings on Privacy Enhancing Technologies 2020, 1 (2020), 195-215. https://doi.org/10.2478/popets-2020-0011

[65] Chad Spensky, Jeffrey Stewart, Arkady Yerukhimovich, Richard Shay, Ari Trachtenberg, Rick Housley, and Robert K. Cunningham. 2016. SoK: Privacy on Mobile Devices - It's Complicated. Proceedings on Privacy Enhancing Technologies 2016, 3 (2016), 96-116. https://doi.org/10.1515/popets-2016-0018

[66] Anna Squicciarini, Cornelia Caragea, and Rahul Balakavi. 2017. Toward Automated Online Photo Privacy. ACM Trans. Web 11, 1, Article 2 (April 2017), 29 pages. https://doi.org/10.1145/2983644

[67] Anna Cinzia Squicciarini, Mohamed Shehab, and Federica Paci. 2009. Collective Privacy Management in Social Networks. In Proceedings of the 18th International Conference on World Wide Web (Madrid, Spain) (WWW'09). Association for Computing Machinery, New York, NY, USA, 521-530. https://doi.org/10.1145/1526709.1526780

[68] Jose M. Such and Natalia Criado. 2016. Resolving Multi-Party Privacy Conflicts in Social Media. IEEE Transactions on Knowledge and Data Engineering 28, 7 (2016), 1851-1863. https://doi.org/10.1109/TKDE.2016.2539165

[69] Jose M. Such and Natalia Criado. 2018. Multiparty Privacy in Social Media. Commun. ACM 61, 8 (July 2018), 74-81. https://doi.org/10.1145/3208039

[70] Jose M. Such, Joel Porter, Sören Preibusch, and Adam Joinson. 2017. Photo Privacy Conflicts in Social Media: A Large-Scale Empirical Study. In Proceedings of the 2017 CHI Conference on Human Factors in Computing Systems (CHI '17). Association for Computing Machinery, New York, NY, USA, 3821-3832. https://doi.org/10.1145/3025453.3025668

[71] Iraklis Symeonidis, Fatemeh Shirazi, Gergely Biczók, Cristina Pérez-Solà, and Bart Preneel. 2016. Collateral Damage of Facebook Apps: Friends, Providers, and Privacy Interdependence. In ICT Systems Security and Privacy Protection, Jaap-Henk Hoepman and Stefan Katzenbeisser (Eds.). Springer International Publishing, Cham, 194-208. https: //doi.org/10.1007/978-3-319-33630-5_14

[72] Emmanuel Syrmoudis, Stefan Mager, Sophie Kuebler-Wachendorff, Paul Pizzinini, Jens Grossklags, and Johann Kranz. 2021. Data Portability between Online Services: An Empirical Analysis on the Effectiveness of GDPR Art. 20. Proceedings on Privacy Enhancing Technologies 2021, 3 (2021), 351-372. https://doi.org/10.2478/popets-2021-0051

[73] Kurt Thomas, Chris Grier, and David M. Nicol. 2010. unFriendly: Multi-party Privacy Risks in Social Networks. In Privacy Enhancing Technologies (PETS 2010. Lecture Notes in Computer Science, Vol. 6205), Mikhail J. Atallah and Nicholas J. Hopper (Eds.). Springer Berlin Heidelberg, Berlin, Heidelberg, 236-252. https://doi.org/10.1007/978-3-642-14527-8_14

[74] Ashwini Tonge, Cornelia Caragea, and Anna Squicciarini. 2018. Uncovering Scene Context for Predicting Privacy of Online Shared Images. Proceedings of the Thirty-Second AAAI Conference on Artificial Intelligence 32, 1 (Apr. 2018), 8167-8168. https://ojs.aaai.org/index.php/AAAI/article/view/12180

[75] Lynn Tsai, Primal Wijesekera, Joel Reardon, Irwin Reyes, Serge Egelman, David Wagner, Nathan Good, and Jung-Wei Chen. 2017. Turtle Guard: Helping Android Users Apply Contextual Privacy Preferences. In Thirteenth Symposium on Usable Privacy and Security (SOUPS 2017). USENIX Association, Santa Clara, CA, 145-162. https://www.usenix.org/ conference/soups2017/technical-sessions/presentation/tsai

[76] Kasisomayajula Viswanath and John R. Finnegan Jr. 1996. The Knowledge Gap Hypothesis: Twenty-Five Years Later Annals of the International Communication Association 19, 1 (1996), 187-228. https://doi.org/10.1080/23808985.1996. 11678931

[77] Na Wang, Jens Grossklags, and Heng Xu. 2013. An Online Experiment of Privacy Authorization Dialogues for Social Applications. In Proceedings of the 2013 Conference on Computer Supported Cooperative Work (San Antonio, Texas, USA) (CSCW'13). Association for Computing Machinery, New York, NY, USA, 261-272. https://doi.org/10.1145/2441776 
2441807

[78] Na Wang, Heng Xu, and Jens Grossklags. 2011. Third-Party Apps on Facebook: Privacy and the Illusion of Control. In Proceedings of the 5th ACM Symposium on Computer Human Interaction for Management of Information Technology (Cambridge, Massachusetts) (CHIMIT '11). Association for Computing Machinery, New York, NY, USA, Article 4 , 10 pages. https://doi.org/10.1145/2076444.2076448

[79] Yang Wang, Pedro Giovanni Leon, Kevin Scott, Xiaoxuan Chen, Alessandro Acquisti, and Lorrie Faith Cranor. 2013. Privacy Nudges for Social Media: An Exploratory Facebook Study. In Proceedings of the 22nd International Conference on World Wide Web (Rio de Janeiro, Brazil) (WWW'13 Companion). Association for Computing Machinery, New York, NY, USA, 763-770. https://doi.org/10.1145/2487788.2488038

[80] Jake Weidman, William Aurite, and Jens Grossklags. 2019. On Sharing Intentions, and Personal and Interdependent Privacy Considerations for Genetic Data: A Vignette Study. IEEE/ACM Transactions on Computational Biology and Bioinformatics 16, 4 (2019), 1349-1361. https://doi.org/10.1109/TCBB.2018.2854785

[81] Dominik Wermke, Nicolas Huaman, Christian Stransky, Niklas Busch, Yasemin Acar, and Sascha Fahl. 2020. Cloudy with a Chance of Misconceptions: Exploring Users' Perceptions and Expectations of Security and Privacy in Cloud Office Suites. In Sixteenth Symposium on Usable Privacy and Security (SOUPS 2020). USENIX Association, 359-377. https://www.usenix.org/conference/soups2020/presentation/wermke

[82] Primal Wijesekera, Arjun Baokar, Lynn Tsai, Joel Reardon, Serge Egelman, David Wagner, and Konstantin Beznosov. 2017. The Feasibility of Dynamically Granted Permissions: Aligning Mobile Privacy with User Preferences. In 2017 IEEE Symposium on Security and Privacy (San Jose, CA, USA) (IEEE S\&P '17). Institute of Electrical and Electronics Engineers, 1077-1093. https://doi.org/10.1109/SP.2017.51

[83] Jakob Wirth, Christian Maier, and Sven Laumer. 2018. The Influence of Resignation on the Privacy Calculus in the Context of Social Networking Sites: An Empirical Analysis. In Proceedings of the Twenty-Sixth European Conference on Information Systems (Portsmouth, UK) (ECIS 2018 Research Papers). Article 161, 16 pages. https://aisel.aisnet.org/ ecis2018_rp/161

[84] Pamela Wisniewski, Muhammad Irtaza Safi, Sameer Patil, and Xinru Page. 2020. Predicting Smartphone Locationsharing Decisions through Self-reflection on Past Privacy Behavior. fournal of Cybersecurity 6, 1 (09 2020), 16 pages. https://doi.org/10.1093/cybsec/tyaa014

[85] Eric Zeng and Franziska Roesner. 2019. Understanding and Improving Security and Privacy in Multi-User Smart Homes: A Design Exploration and In-Home User Study. In 28th USENIX Security Symposium (USENIX Security '19). USENIX Association, Santa Clara, CA, 159-176. https://www.usenix.org/conference/usenixsecurity19/presentation/zeng

Received January 2021; revised April 2021; accepted July 2021. 


\section{A OPEN-ENDED RESPONSES}

The following open-ended responses entered by the participants in response to the question "Is there anything else you would like to tell us?" were connected to the content of the study.

- I was already concerned about my privacy when it comes to apps. The study made me even more aware of what is being shared with them. Thank you for the opportunity to participate.

- Smartphones are basically spy devices that we were all suckered into buying and now basically need in our daily lives. Meanwhile the camera and microphone can be accessed at any time by the NSA and god knows who else. Knowing this, I keep as little personal info on my phone as I can and use it only sparingly... and begrudgingly because land lines have basically gone the way of the Dodo.

- I was really surprised when I looked at how many apps are actually installed on my phone when I looked for the exact number. Thank you.

- I usually try to avoid any apps that ask for permissions.

- Due to increasing privacy concerns, I have recently switched to as much use of a dumb phone as possible. On my smartphone, I try to limit app downloads, use a private browser, and turn off tracking like location as much as possible.

- I learned something new today - that I have way more apps on my phone than I thought! Time to verify if I really NEED them and to get as much private information off of my phone as I can to store another way (thumb drive) ASAP. It's disturbing that so much of our personal information is out there for the taking. Thank you for reminding us of this fact!

- Important study. I like the awareness.

- I learned something from this study.

- I rarely use the calendar, only for major events.

- I store my photos on Google photos in the cloud, not my phone locally.

- My number of calendar entries is reported as zero because I don't keep appointments and other data on my smartphone. I have a paper calendar for that.

- I think that it is pretty worrying when an app is asking me for personal access to my information which could be used to spy on me or provide compromising information to a server provider.

- I actually really enjoyed this study. I knew apps asked access to Contacts. With those apps, I am just a little more hesitant with installing them. However, I normally read reviews on apps, considering all my apps are for work related-purposes. I had a little nauseous feeling when seeing how many apps I actually have on my phone. I had the same nauseous feeling with the photos, since that is what $50 \%$ of my apps ask access to. This study definitely made me more aware, since I do have photos of my family as well as other photos too. I did not think I had anywhere near that many photos in my phone. I definitely want to say thank you for the study. I hope you all have a great day and a great week!

- You have a question that asks participants how much they'd consider paying to use a paid version of the app to prevent access to the Calendar, but you never specified what the app is actually for. Not knowing why Thomas wanted the app for in the first place, it's hard to put a value on it. Privacy-conscious people wouldn't use the app to begin with, so they wouldn't put a monetary value on the app at all.

- Interesting, thanks. Gives a person a lot to think about!

- I have a degree in networking \& system administration and have worked in tech support and the telecom industry. I am well aware of data collection and their invasion of our privacy which is why I generally install few apps. Currently, only running a secure messaging app, 
work app, and a security camera app beyond what was installed by default. I especially avoid large corporations apps, such as Facebook and Google for instance.

- I feel pretty ignorant after this study. Thanks for enlightening me about the possibilities of abuse.

- I do enjoy using my smartphone. It makes my life easier. However, I try my hardest to not share too much with applications because I do not feel comfortable sharing so much information. I am not even all that active on social media anymore.

- That was a lot of questions, but interesting. I am shocked by the number of apps I have.

- I disable a lot of standard apps on my phone. I use an email only for Google Play strictly for phone apps and not my regular email for privacy concerns.

- This was a very interesting task to think about and complete. I am always interested in my own as well as others' privacy when using apps and websites online.

- There is no free lunch. I have a number of daily reminder CM entries that stay till I get something finished.

- In regard to the very first part of the survey: you didn't say WHAT app asked for access to his photos. If it's a photo-related app, then it would make complete sense, and I probably wouldn't give too much thought to installing it after a quick Google search to make sure it's on the up-and-up. If it was an app that had nothing to do with photos, however, that would raise all kinds of red flags for me, and I probably would uninstall it immediately. The context there is really important to my answers. Also, I hope you have a great day!

- For clarification, I recently acquired my phone, so I have very few apps and no contacts.

- Don't trust any company.

- I thought I only had about 20 apps, I was shocked I had 64.

- Our data is being collected for destructive purposes.

- This study has made me question my decisions to allow apps to access various functions on my phone. I don't like it when they require such access, but oftentimes I go ahead and download it anyways.

- I don't keep photos on my phone because of privacy issues. I thought I had discarded the two photos on my phone but when I looked, when the question was asked in the study, I realized they were still in my cloud. I try to keep as much personal info off my phone as possible. I allow Google to access my personal info so it will link all my devices, in addition to apps I use frequently, but am suspicious of new apps I install. I have never thought about the privacy of my friends. I receive ads based on my browsing history, not my friends, so I would think they don't receive ads based on my browsing history. Although, who knows. I receive calls from telemarketers related to things I have never searched and app suggestions that don't match my likes nor history.

- Privacy is important, but it is hard to handle in this 21st century digital age!

- I liked the way of questions. Thanks. You helped me out to know the storage of my phone and know some techniques.

- It's an up-to-date study to know better about mobile apps which is quite important.

- I didn't have my phone with me to check the exact numbers requested, so I just roughly guessed. 


\section{B STUDY INSTRUMENT}

\section{B.1 Study Information}

You are being invited to participate in a research study titled "Questionnaire on Smartphone Usage." This study is being done by researchers from the Technical University of Munich. [CONTACT INFORMATION]

Purpose of this study: In this study, we aim to investigate the differences in smartphone usage.

Procedures to be followed: To complete the study, you will be asked to answer several questions. At the end, you will get a personal code that you can use to get the payment for completing the study. Please enter the code on Amazon Mechanical Turk.

Discomforts and risks: There are no risks in participating in this study beyond those experienced in everyday life. Questionnaire responses and your behavior during the survey will not be used to identify you in any way.

Duration/Time: The whole process should take you about 10 minutes on average.

Statements of confidentially: Your participation in this research is confidential. This study does not ask for any information that would identify you based on your responses. In the event of any publication or presentation resulting from the research, no personally identifiable information will be shared because your name is in no way linked to your responses.

Right to ask questions: Please contact [CONTACT INFORMATION] with questions, complains or concerns about this research. You can use the contact information if you feel this study has harmed you.

Payment for participation: You will receive compensation via Mechanical Turk.

Voluntary participation: Your decision to participate in this research study is voluntary. You can stop any time. You do not have to answer any questions you do not want to answer.

Completion of this study implies that you have read the above information and consent to take part in this research.

By clicking "I agree" below you are indicating that you are at least 18 years old, have read and understood the above information, and agree to participate in this research study.

- No, I do not agree (do not participate in this study).

- Yes, I agree.

\section{B.2 Scenario Description}

Imagine the following hypothetical scenario:

Thomas is an undergraduate student at his local University. He is looking for a new app for his smartphone. After some searching, he found an app which he installed on his phone without hesitation. 
This app asked for access to the [calendar OR contacts OR photos], which Thomas granted.

In the following, you can see a screenshot of his [calendar OR contacts OR photos] (See Figures C.1, C.2, C.3 and C.4, C.6 and C.5). Have a closer look at his [calendar OR contacts PR photos].

In the background, the application is sending the information stored in the [calendar

OR contacts OR photos] to the server of the app maker.

[SCREENSHOT OF CALENDAR OR CONTACTS OR PHOTOS, EITHER NON-PERSON OR PERSON]

\section{B.3 Questionnaire}

- [MANIPULATION CHECK 1:] What kind of [calendar events/contacts/photos] does Thomas have?

Think of what you saw in the previous picture, and choose the correct answer.

- CALENDAR:

○ Mainly listings of events (birthdays, parties, holidays, etc.)

- Mainly listings of lectures

- CONTACTS:

- Mainly family and friends

- Mainly companies and institutions

- PHOTOS:

- Mainly photos from family/friends/other people

- Mainly photos from landscapes and cities

[If the response is incorrect, show the participant the following text: The option you chose was wrong.

CALENDAR: Thomas has mainly calendar events of [listings of events (birthdays, parties, holidays, etc.) / listings of lectures] on his phone.

CONTACTS: Thomas has mainly contacts of [family and friends / companies and institutions] on his phone.

PHOTOS: Thomas has mainly photos of [family, friends, and other people / landscapes and cities] on his phone. ]

- [MANIPULATION CHECK 2:] What permission did the app ask for?

- Access to Calendar

- Access to Contacts

- Access to Photos

[If the response was incorrect, show the participant the following text: The option you chose was wrong.

CALENDAR: The app asked for access to calendar.

CONTACTS: The app asked for access to contacts.

PHOTOS: The app asked for access to photos. ]

- Installing this app... [5-point scale from 1 = Strongly Disagree to 5 = Strongly Agree]

- ...could jeopardize Thomas's privacy.

- ... would result in a high potential loss of privacy for Thomas. 
- ... could lead to inappropriate use of Thomas's data.

- Installing this app... [5-point scale from 1 = Strongly Disagree to $5=$ Strongly Agree]

- ... could jeopardize the privacy of Thomas's friends.

- ...would result in a high potential loss of privacy for Thomas's friends.

- ...could lead to inappropriate use of Thomas friends' data.

- Thomas is responsible for protecting friends' data which is stored on his device. [5-point scale from 1 = Strongly Disagree to 5 = Strongly Agree]

- Third parties are responsible for protecting friends' which is stored on Thomas's device. [5-point scale from 1 = Strongly Disagree to 5 = Strongly Agree ]

- Assuming you were Thomas and knowing that this app has access to Thomas's [calendar OR contacts OR photos]: [5-point scale from $1=$ Strongly Disagree to $5=$ Strongly Agree]

- I would be willing to install this app.

- It is likely that I would install this app.

- Assume again that you are Thomas. You have the chance to prevent access to your [calendar OR contacts OR photos] by using a paid version of the app instead of a free one. Both versions of the app provide the same functionality.

What is the maximum amount of money you would be willing to spend in order to install the paid version of the app? [Text box]

- The following questions are NOT based on the scenario above:

Please answer the questions from your own perspective. [5-point scale from 1 = Strongly Disagree to 5 = Strongly Agree]

- Granting an app access to my calendar has the potential for revealing privacy-sensitive content.

- Granting an app access to my contacts has the potential for revealing privacy-sensitive content.

- Granting an app access to my photos has the potential for revealing privacy-sensitive content.

- I feel comfortable granting an app access to my calendar.

- I feel comfortable granting an app access to my contacts.

- I feel comfortable granting an app access to my photos.

- If an app asks to access calendar, contacts, and photos, you cannot restrict access to only part of your calendar events, contacts, or photos. This app then has the possibility to read all of your calendar events, contacts, and photos and transfer those to the server of a third party. Are you aware of this?

- Yes

- No

- Please answer the following questions about your device usage.

- How many apps do you have installed on your phone at the moment? [INSTRUCTIONS AND SCREENSHOTS FOR HOW TO OBTAIN THE INFORMATION ON ANDROID AND iOS] [Text box]

- How many photos do you have on your phone? [INSTRUCTIONS AND SCREENSHOTS FOR HOW TO OBTAIN THE INFORMATION ON ANDROID AND iOS] [Text box] 
- Did you approximate the number of photos?

- I approximated the number of photos.

- I gave the exact number of photos.

- How many contacts do you have on your phone? [Text box]

- Did you approximate the number of contacts?

- I approximated the number of contacts.

$\circ$ I gave the exact number of contacts.

- For a typical week, how many private/family-related calendar events do you have on your phone? [Text box]

- For a typical week, how many work/school-related calendar events do you have on your phone? [Text box]

- Please indicate your level of agreement with each of the following statements: [5-point scale from 1 = Strongly Disagree to 5 = Strongly Agree]

- Companies today have the ability to place online advertisements that target you based on information collected about your web browsing behavior.

- When you go to a website, it can collect information about you even if you do not register.

- Popular search engine sites, such as Google, track the sites you come from and go to.

- Many of the most popular third-party apps reveal users' information to other parties, such as advertising and Internet tracking companies.

- I have recently helped a person with a problem.

- I should go out of my way to help people more often.

- If a member of my 'social group' comes to me with a personal problem, I'm willing to listen without being judgmental.

- If a member of my 'social group' needs help on a task, I am willing to help even if it causes me some inconvenience.

- I am willing to help a 'social group' member I don't know.

- Please be aware that the following questions are about your personal information. [5-point scale from 1 = Strongly Disagree to 5 = Strongly Agree]

- It usually bothers me when third-party app developers ask me for personal information.

- When third-party app developers ask me for personal information, I sometimes think twice before providing it.

- It bothers me to give my personal information to so many third-party app developers.

- I'm concerned that third-party app developers are collecting too much personal information about me.

- Please be aware that the following questions are about personal information of your friends. [5-point scale from $1=$ Strongly Disagree to 5 = Strongly Agree]

- It usually bothers me when third-party app developers ask me for my friends' personal information.

- When third-party app developers ask me for my friends' personal information, I sometimes think twice before providing it.

- It bothers me to give my friends' personal information to so many third-party app developers. 
- I'm concerned that third-party app developers are collecting too much personal information about my friends.

- What is your gender?

- Male

- Female

- Something else

- Prefer not to say

- If you chose 'Something else' then please specify your gender: [Text box]

- What is your age?

$-<18$

$-18-24$

$-25-34$

$-35-44$

$-45-54$

$-55-64$

$-65+$

- What is the highest level of education you have completed?

- No school completed

- Primary school

- Some high school, no diploma

- Some collage credit, no degree

- Bachelor's degree

- Master's degree

- Doctoral degree

- Something else

- Prefer not to answer

- If you chose 'Something else' then please specify your level of education:. [Text box]

- What is your annual income?

- $\$ 0$

- $\$ 1$ to $\$ 9.999$

- $\$ 10.000$ to $\$ 24.999$

- $\$ 25.000$ to $\$ 49.999$

- $\$ 50.000$ to $\$ 74.999$

- $\$ 75.000$ to $\$ 99.999$

- $\$ 100.000$ to $\$ 149.999$

- $\$ 150.000$ and greater

- How long have you lived in the United States?

- less than 1 year

-1 year 
-2 years

-3 years

-4 years

-5 years

- 6 years or more / my whole life

- What the sum of three and four? [ATTENTION CHECK QUESTION]

$-4$

$-5$

$-6$

$-7$

$-8$

$-9$

- What is your martial status?

- Single, never married

- Married

- Widowed

- Divorced

- Separated

- Prefer not to answer

- Do you have children?

- No

- Yes, 1 child

- Yes, 2 children

- Yes, 3 children

- Yes, 4 children

- Yes, more than 4 children

- Prefer not to answer

- Which operating system do you use?

- Android

- iOS

- Other

- Is there anything else you would like to tell us? [Text Box]

Thank you for completing this questionnaire.

Your personal code for getting the reward on Amazon Mechanical Turk is: [RANDOMLY-GENERATED UNIQUE CODE]

Please copy the code before closing this tab.

Thank you for taking the time to participate in the study. 


\section{VISUAL DEPICTIONS USED IN THE STUDY}

\begin{tabular}{|c|c|c|c|c|c|c|c|c|c|c|}
\hline & Mon & & Tue & & Wed & & Thu & Fri & Sat & Sun \\
\hline 44 & 28 & & 29 & & 30 & & 31 & 1. Nov & 2 & 3 \\
\hline - Lecture Managem... & $15: 00$ & - Lecture Digitalisati... & $16: 00$ & $\begin{array}{l}\text { - Lecture Marketing } \\
\text { - Lecture Accounting }\end{array}$ & $\begin{array}{l}09: 00 \\
11: 00\end{array}$ & $\begin{array}{l}\text { - Lecture Korean } \\
\text { Exercise Analysis }\end{array}$ & $\begin{array}{l}11: 30 \\
15: 00\end{array}$ & - Lecture Marketing $\quad 09: 00$ & & \\
\hline 45 & 4 & & 5 & & 6 & & 7 & 8 & 9 & 10 \\
\hline $\begin{array}{l}\text { Guest Speaker Am... } \\
\text { - Lecture Managem... }\end{array}$ & $\begin{array}{l}10: 00 \\
15: 00\end{array}$ & $\begin{array}{l}\text { - Lecture Analysis } \\
\text { Lecture Digitalisati... }\end{array}$ & $\begin{array}{l}09: 00 \\
16: 00\end{array}$ & $\begin{array}{l}\text { - Lecture Marketing } \\
\text { - Lecture Accounting }\end{array}$ & $\begin{array}{l}09: 00 \\
11: 00\end{array}$ & $\begin{array}{l}\text { - Lecture Korean } \\
\text { Exercise Analysis }\end{array}$ & $\begin{array}{l}11: 30 \\
15: 00\end{array}$ & - Lecture Marketing $\quad 09: 00$ & & \\
\hline 46 & 11 & & 12 & & 13 & & 14 & 15 & 16 & 17 \\
\hline - Lecture Managem... & $15: 00$ & $\begin{array}{l}\text { - Lecture Analysis } \\
\text { - Lecture Digitalisati... }\end{array}$ & $\begin{array}{l}09: 00 \\
16: 00\end{array}$ & $\begin{array}{l}\text { - Lecture Marketing } \\
\text { - Lecture Accounting } \\
\text { - Guest Speaker Mic... }\end{array}$ & $\begin{array}{r}09: 00 \\
11: 00 \\
15: 30\end{array}$ & $\begin{array}{l}\text { - Lecture Korean } \\
\text { - Exercise Analysis }\end{array}$ & $\begin{array}{l}11: 30 \\
15: 00\end{array}$ & - Lecture Marketing $\quad 09: 00$ & & \\
\hline 47 & 18 & & 19 & & 20 & & 21 & 22 & 23 & 24 \\
\hline - Lecture Managem... & $15: 00$ & $\begin{array}{l}\text { - Lecture Analysis } \\
\text { - Lecture Digitalisati... }\end{array}$ & $\begin{array}{r}09: 00 \\
16: 00\end{array}$ & $\begin{array}{l}\text { - Lecture Marketing } \\
\text { - Lecture Accounting }\end{array}$ & $\begin{array}{l}09: 00 \\
11: 00\end{array}$ & $\begin{array}{l}\text { - Lecture Korean } \\
\text { - Exercise Analysis }\end{array}$ & $\begin{array}{l}11: 30 \\
15: 00\end{array}$ & - Lecture Marketing $\quad 09: 00$ & & \\
\hline 48 & 25 & & 26 & & 27 & & 28 & 29 & 30 & 1. Dec \\
\hline - Lecture Managem... & $15: 00$ & $\begin{array}{l}\text { - Lecture Analysis } \\
\text { - Lecture Digitalisati... }\end{array}$ & $\begin{array}{l}09: 00 \\
16: 00\end{array}$ & $\begin{array}{l}\text { - Lecture Marketing } \\
\text { - Lecture Accounting }\end{array}$ & $\begin{array}{l}09: 00 \\
11: 00\end{array}$ & $\begin{array}{l}\text { - Lecture Korean } \\
\text { - Exercise Analysis }\end{array}$ & $\begin{array}{l}11: 30 \\
15: 00\end{array}$ & & & \\
\hline
\end{tabular}

Fig. C.1. Calendar visual depiction used in the Non-persons condition.

\begin{tabular}{|c|c|c|c|c|c|c|c|}
\hline 1. Jul & & 2 & 3 & 4 & 5 & 6 & 7 \\
\hline \multirow[t]{2}{*}{ Toni on conference in LA } & & & & & \multirow{2}{*}{$\begin{array}{l}\text { Mia Birthday } \\
\text { Max first day - new job }\end{array}$} & \multirow[t]{2}{*}{ Party with Paul Max Finn... } & \multirow{2}{*}{$\begin{array}{l}\text { Marc on holiday in Spain } \\
\text { Dinner with family }\end{array}$} \\
\hline & $\begin{array}{l}\text { Tennis with Luca } \\
\text { Dinner with Julia }\end{array}$ & $\begin{array}{l}09: 00 \\
19: 00\end{array}$ & $\begin{array}{lr}\text { - Meeting with Joe } & 14: 00 \\
\text { - Cinema with Max P... 20:00 }\end{array}$ & & & & \\
\hline 8 & & 9 & 10 & 11 & \multirow{4}{*}{$\begin{array}{r}12 \\
\text { Move Patrik Berlin - Mun... }\end{array}$} & \multirow[t]{4}{*}{13} & \multirow{4}{*}{ Lisa and Eva on holiday i... } \\
\hline Marc on holiday in Spain & & & Kevin in Munich & \multirow{3}{*}{ Austria wellness with Julia } & & & \\
\hline \multirow[t]{2}{*}{ - Meeting with Tim OB:00 } & Johannes Birthday & & - Pick Up Franz from... 16:30 & & & & \\
\hline & - Tennis with Luca & $09: 00$ & - Dinner with Fritz $\quad 19: 00$ & & & & \\
\hline 15 & & 16 & 17 & 18 & 19 & \multirow[t]{3}{*}{20} & 21 \\
\hline \multicolumn{7}{|c|}{ Lisa and Eva on holiday in Korea } & \\
\hline \multirow[t]{2}{*}{ 4. Wedding day Hannes... } & Tennis with Luca & $09: 00$ & Till in Munich & & \multirow[b]{2}{*}{ Zoo with Cousin Felix } & & \multirow{2}{*}{$\begin{array}{l}\text { water nighbours' plants... } \\
\text { Wedding Ella and Levin }\end{array}$} \\
\hline & Visit Paul in Berlin & $17: 00$ & ends 18:00 & Party with Lina Lisa Ben... & & Julia away with friends & \\
\hline 22 & & 23 & 24 & 25 & 26 & \multirow[t]{4}{*}{27} & \multirow{4}{*}{ Barbecue with Jakob } \\
\hline Lisa and Eva on holiday i... & Tennis with Luca & $09: 00$ & Holiday Ella and Levin South & Afrika & & & \\
\hline water nighbours' plants - ho & liday in Italy & & & & & & \\
\hline Dinner with Julia & & & & Jenna Birthday & Help Leon shop for new... & & \\
\hline 31 & & 30 & 31 & 1. Aug & 2 & 3 & 4 \\
\hline $\begin{array}{l}\text { Holiday Ella and Levin S... } \\
\text { Hiking with Lukas }\end{array}$ & $\begin{array}{l}\text { Mom Birthday } \\
\text { Tennis with Luca }\end{array}$ & 09:00 & & & & & \\
\hline & · Tennis with Luca & 09:00 & & & & & \\
\hline
\end{tabular}

Fig. C.2. Calendar visual depiction used in the Persons condition. 


\begin{tabular}{|c|c|c|}
\hline Name & Email & Phone number \\
\hline CONTACTS (62) & & \\
\hline ADAC & info@adac.de & +498005101112 \\
\hline AOK healthinsurance & info@aok.de & +499131924210128 \\
\hline Deutsche Bank & info@dbank.de & 1 (212) $250-2500$ \\
\hline Eon & info@eon.com & +4918832818271 \\
\hline Hanna Luus (Travel agency) & info@travel.com & +4970007000 \\
\hline HUK car insurance & info@huk.de & +498002485445 \\
\hline ING (Bank) & info@ing.com & +496950500105 \\
\hline Leon Allock (Facility Management) & info@allock.com & +12069220880 \\
\hline Luftansa & info@luftansa.de & +4917727221 \\
\hline Maja Linde (Gardener) & info@linde.de & +494977172 \\
\hline Oliver Ambridge (Family doctor) & info@ambridge.com & +498006645451 \\
\hline Telekom & info@telekom.de & +498003301000 \\
\hline United & info@united.de & +80033552247 \\
\hline Visa & info@visa.com & +4918008472911 \\
\hline
\end{tabular}

Fig. C.3. Contacts visual depiction used in the Non-persons condition. 


\begin{tabular}{|c|c|c|c|}
\hline \multirow{2}{*}{$\begin{array}{l}\text { Name } \\
\text { MYFRIENDS (62) }\end{array}$} & Email & Phone number & Birthday \\
\hline & & & \\
\hline Anna Alvar & ealvarnj@ftc.gov & +496668548543 & $12 / 16 / 1979$ \\
\hline Dad & franky@over-blog.com & +494527415871 & $10 / 23 / 1972$ \\
\hline Grandma & gran@1und1.de & +492062142882 & $5 / 21 / 1941$ \\
\hline Grandpa & gran@1und1.de & +495063694927 & $10 / 16 / 1940$ \\
\hline Hanna Luus & callabartonfm@dyndns.org & +495189151110 & $2 / 17 / 1989$ \\
\hline Laura Kloos & laldrich4n@webs.com & +493406776147 & $10 / 25 / 1986$ \\
\hline Leon Steel & hamottdj@ocn.ne.jp & +498903793178 & $4 / 30 / 1977$ \\
\hline Marvin Anders & sandrzejczak5i@sciencedirec... & +492728630237 & $7 / 31 / 1970$ \\
\hline Mom & nad@theatlantic.com & +498053711404 & $2 / 13 / 1975$ \\
\hline Oliver Ambridge & nambridgei6@jalbum.net & +491717756768 & 6/13/1975 \\
\hline Sabine Birke & calmackmr@seesaa.net & +498111706658 & $11 / 13 / 1972$ \\
\hline Sonja Stoos & aanettsjq@naver.com & $106-290-2639$ & $12 / 14 / 1985$ \\
\hline Sven Brown & tandor6r@vk.com & +494205493719 & 4/14/1976 \\
\hline Sweetheart $\vartheta$ & julia@icloud.com & +496615886280 & $4 / 21 / 1998$ \\
\hline
\end{tabular}

Fig. C.4. Contacts visual depiction used in the Persons condition. 
20:18 Wed 22. May

$<$ Albums
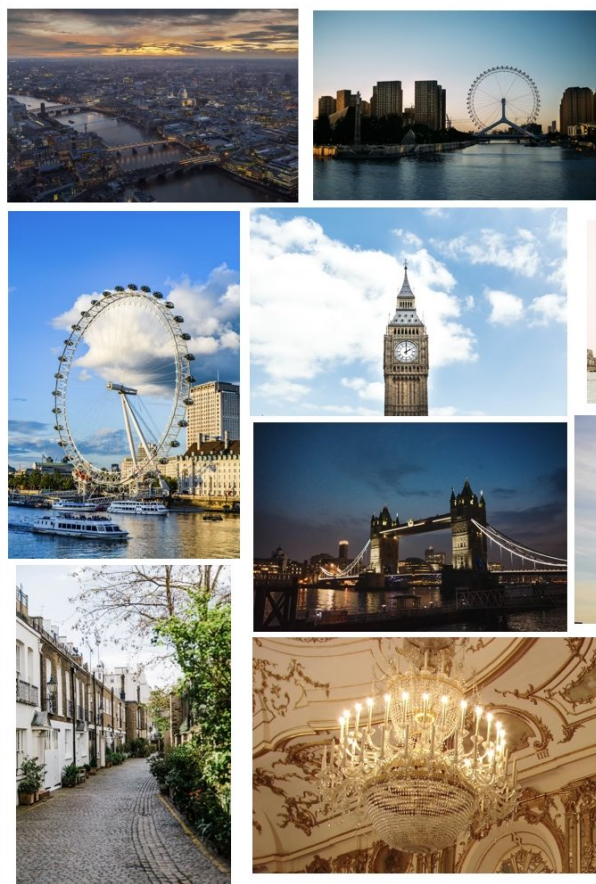

All Photos

II $64 \%$

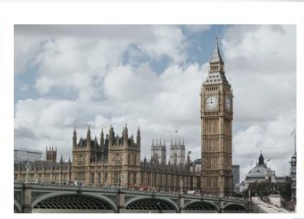
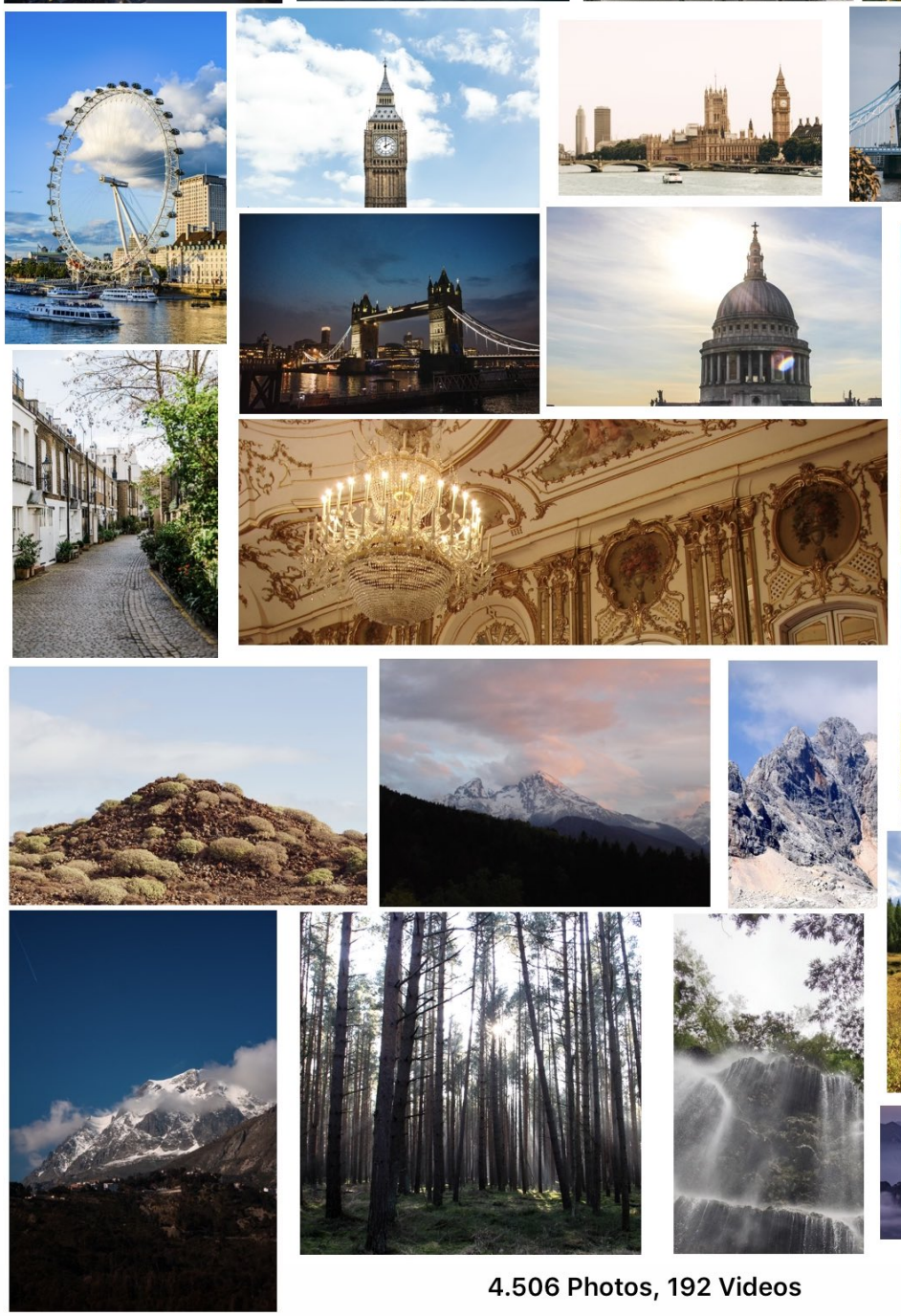

4.506 Photos, 192 Videos
Slideshow
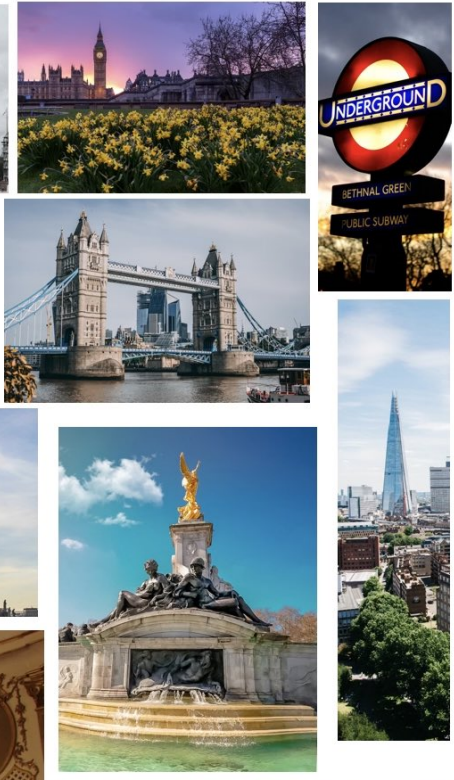

Select 

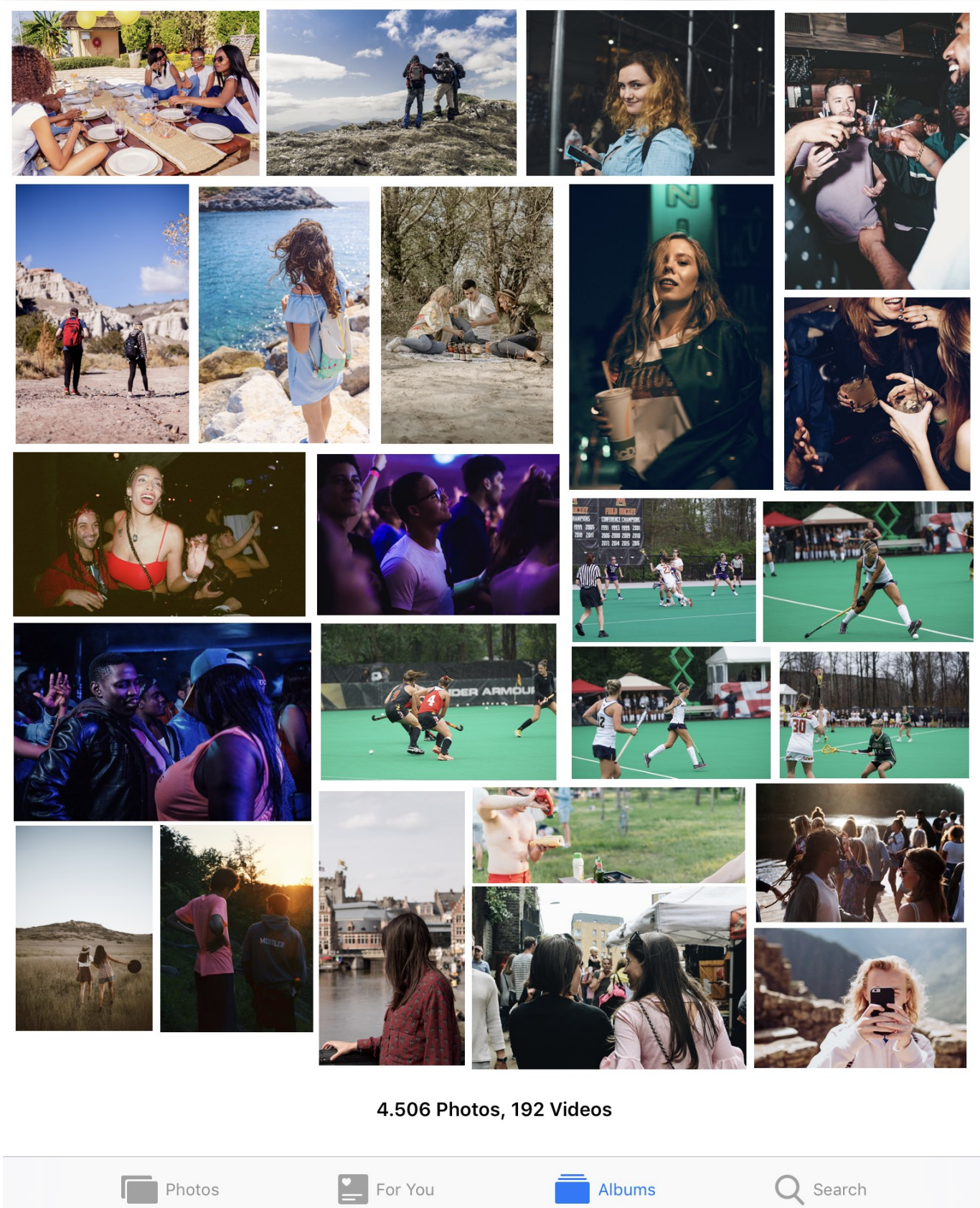

\subsection{Photos, 192 Videos}

Fig. C.6. Photos visual depiction used in the Persons condition. Images taken from https://unsplash.com and used under a free license. 